\title{
KELTISCHE KOSTBARKEITEN AUF DEN KOPF GESTELLT
}

Sophie Hüglin und Norbert Spichtig diskutieren mit Marion Benz über den keltischen Jahrhundertfund von Basel-Gasfabrik 


\section{FUND:}

DEPONIERUNG

\section{DATIERUNG:}

2./1. JH. V. CHR.

\section{FUNDORT:}

\section{BASEL-GASFABRIK}

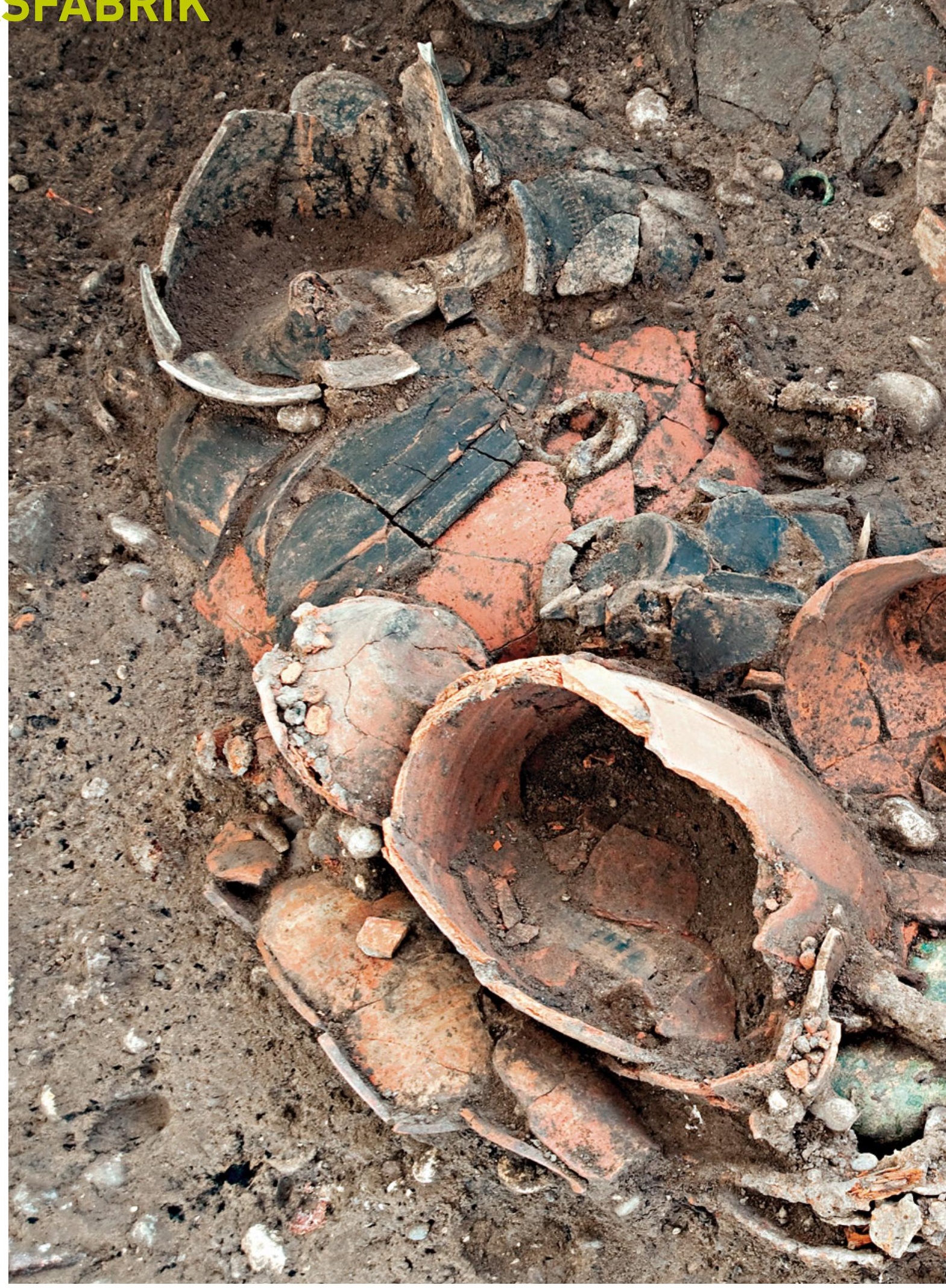


Abb. 1 Einzigartig: Für die keltische

Deponierung von Basel-Gasfabrik

gibt es kaum vergleichbare Befunde

aus der späten Eisenzeit Europas.

Unter einer Schicht qualitätsvoller,

vollständig erhaltener Keramikgefäs-

se zeichnet sich bereits die nächste

Lage mit den grün oxidierten Objek-

ten aus Buntmetall ab. Nur für das

geschulte Auge zu erkennen sind die

Reste der Behältnisse aus Holz.

Foto: Denise Grossenbacher.

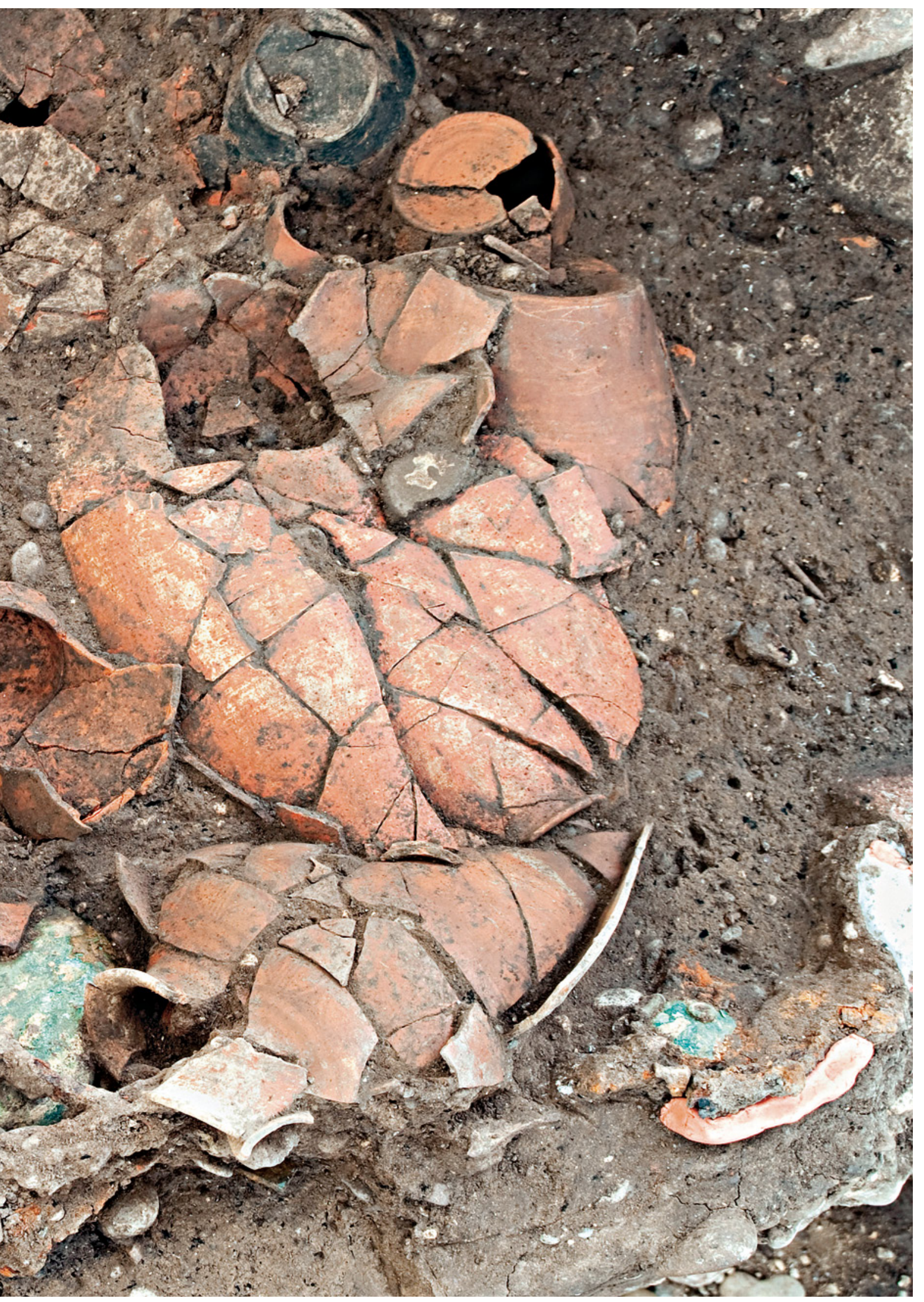




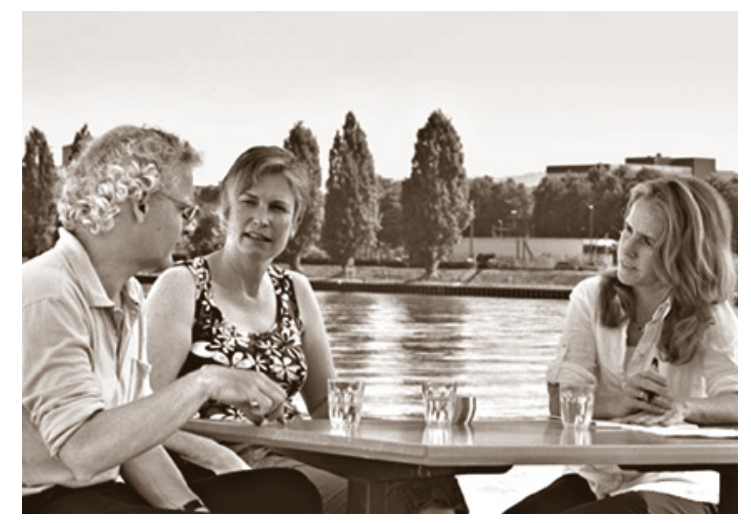

Seit der Entdeckung des keltischen Schatzfunds von Basel-Gasfabrik im Februar 2010 warten Fachwelt und Öffentlichkeit gespannt auf dessen Freilegung. Derzeit werden die wertvollen Stücke unter Laborbedingungen vorsichtig geborgen. Schon jetzt aber erörtern Norbert Spichtig, verantwortlich für das Grossprojekt Basel-Gasfabrik, und Sophie Hüglin, wissenschaftliche Leiterin der Grabung, den Jahrhundertfund. In einem Punkt sind sie sich einig: Das Depot ist bislang einzigartig; über manch anderes lässt sich jedoch trefflich streiten.

Norbert Spichtig - der bereits seine Lizentiatsarbeit über BaselGasfabrik geschrieben hat - kennt die Fundstelle wie seine Westentasche. Seit über 20 Jahren arbeitet er bei der Archäologischen Bodenforschung Basel-Stadt (ABBS). 1999 wurde er Leiter des Ressorts Gasfabrik und im Jahr darauf stellvertretender Kantonsarchäologe. Sein Interesse an modernsten digitalen Dokumentationstechniken erweist sich jetzt als Glücksfall für die Deponierung.

Sophie Hüglin bringt die Aussenperspektive ein. Schon während ihres Studiums in Freiburg im Breisgau gilt ihre Leidenschaft den Kelten. Sie lernt Irisch, liest alt-irische Literatur und erforscht keltische Ortsnamen. 1988/89 studiert sie in Irland. Nach ihrer Promotion führt sie verschiedene Ausgrabungen in Süddeutschland durch. 2005 übernimmt sie die Stelle einer wissenschaftlichen Grabungsleiterin bei der ABBS und nur drei Jahre später die stellvertretende Leitung des Ressorts Basel-Gasfabrik. Ihre ideenreichen Thesen zum Depot faszinieren und provozieren zugleich.

Am Fundort: Norbert Spichtig, Sophie Hüglin und Marion Benz (von links nach rechts) beim Interview auf der Grossbaustelle im ehemaligen Rheinhafen St. Johann. Nur wenige Meter entfernt wurde im Sommer 2010 das einzigartige keltische Depot entdeckt. Foto: Philippe Saurbeck. 
Vor genau 100 Jahren wurde die keltische Siedlung BaselGasfabrik entdeckt. Seitdem wird sie wie kaum eine andere eisenzeitliche Fundstelle intensiv erforscht. Was rechtfertigt diesen enormen Aufwand? War die Siedlung so bedeutend?

NORBERT SPICHTIG Zunächst ist es Auftrag jeder archäologischen Fachstelle, das zu retten, was sonst durch Baumassnahmen zerstört würde. «Mehr oder weniger bedeutend» steht gar nicht so im Vordergrund. Es sind deshalb reine Rettungsgrabungen, die wir hier durchführen. Aber effektiv ist es natürlich so, dass Basel-Gasfabrik in der archäologischen Forschung immer eine grössere Bedeutung gehabt hat. Schon mit Karl Stehlin Anfang des 20. Jahrhunderts fing das an: Er hat seine Ergebnisse schnell publiziert und in der Fachwelt grosse Resonanz gefunden, danach ist es etwas stiller geworden, aber ab Mitte der siebziger Jahre durch das Schweizerische Nationalfonds-Projekt von Ludwig Berger und Andres Furger-Gunti hat die Siedlung wieder einen sehr hohen Stellenwert bekommen. Durch diese Aufarbeitung wurden die Funde und Befunde zu einem chronologischen Fixpunkt, an dem sich andere europäische Forschung orientiert. Nach wie vor strahlt diese Forschung weit über unsere Grenzen aus. Basel-Gasfabrik ist also zu Recht im Kulturgüterverzeichnis der Schweiz als eine Fundstelle von nationaler Bedeutung aufgeführt.

SOPHIE HÜGLIN Ich habe da mehr die Aussenperspektive: da ich nicht in Basel, sondern in Freiburg im Breisgau studiert habe. In den Vorlesungen galten Basel-Gasfabrik und BaselMünsterhügel als die Paradebeispiele für die Siedlungsentwicklung am Ende der Eisenzeit: offene unbefestigte Grosssiedlungen in der Ebene wie Basel-Gasfabrik werden abgelöst von befestigten Siedlungen auf Anhöhen. Dieser Prozess ist an verschiedenen Orten in Mitteleuropa zu beobachten, z.B. auch in Breisach. Ich erinnere mich noch gut, dass mein Professor uns damals vorrechnete, dass der Fundort Basel-Gasfabrik, der nur über ca. drei Generationen besiedelt war, inzwischen schon länger ausgegraben wird, als er bestanden hat. Das ist aber bei vielen anderen bedeutenden - sonst meist römischen - Fundorten mit langer Forschungstradition so. 
Abb. 2 Sorgfältig und vorsichtig, aber so zügig wie möglich legen die Archäologen das keltische Depot frei und dokumentieren es. Schnell merken sie jedoch, dass der Zeitplan so nicht einzuhalten ist. Foto: Michael Wenk.

Abb. 3 Jede freigelegte Schicht wird dreidimensional eingemessen und gescannt. Inzwischen werden mit einer speziellen Software und weniger Aufwand 3D-

Modelle von jeder Schicht erstellt

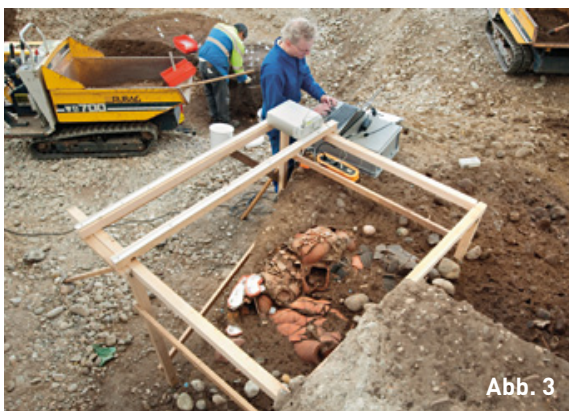

(«Structure-from-Motion»). Foto: Denise

Grossenbacher.

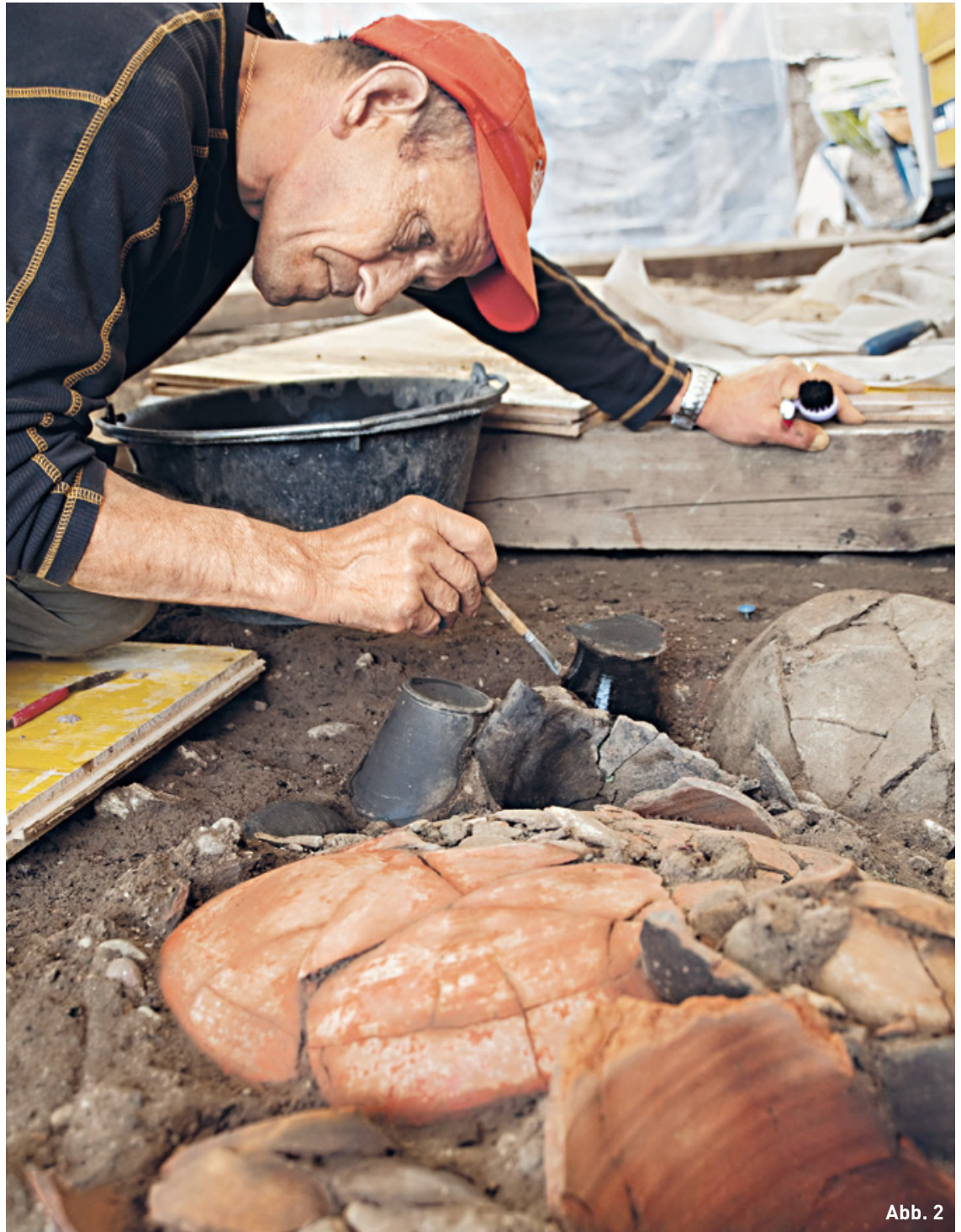


N. SPICHTIG Die aktuelle Forschung hat tatsächlich Hinweise darauf, dass Basel-Gasfabrik schon in der Antike eine zentrale Bedeutung für das Gebiet am südlichen Oberrhein zukam. Aber wie gesagt, für uns steht nicht nur die Einzelfundstelle im Vordergrund. Wir versuchen auch historische Entwicklungen insgesamt zu erforschen: Was war an diesem Ort und wie ist das einzuordnen?

S. HÜGLIN So überraschend es klingt, aber die vielen Grabungen in den letzten 20 Jahren haben sogar teilweise verhindert, dass es bislang zu einer umfassenden Auswertung kam.

N. SPICHTIG Ja, unsere begrenzten Kapazitäten sind durch die Feldarbeiten für die mittlerweile vier Grossbauprojekte seit 1988 stark gebunden worden. Das fing mit den «Sandoz»Grabungen an, dann kamen die Grabungen für die Nordtangenten-Autobahn, die zeigten, dass die Siedlung grösser war als gedacht. Noch während die Grabungen an der Nordtangente liefen, begann Novartis damit, ihr Campus-Grossprojekt umzusetzen, und jetzt wird der ehemalige Rheinhafen St. Johann zum «Campus plus» umgestaltet. Das war alles so nicht absehbar und wir sind selbst immer wieder überrascht worden.

Letztes Jahr habt ihr das sensationelle Depot entdeckt (s. Vorbericht zur Grabung 2009/36 in: Ausgrabungen und Funde im Jahr 2010, Gasfabrik). Wenn aber ein so hoher Zeitdruck besteht, ist ein solcher Jahrhundertfund dann nicht eher eine Katastrophe, weil er alles aufhält?

N. SPICHTIG Nein, eine Katastrophe ist er sicher nicht. Im Gegenteil! Man muss konstruktiv damit umgehen. Mehr Finanzen bekommen wir nicht, so dass die Aufwendungen durch Umlagerungen von anderen Posten aufgefangen werden müssen.

S. HÜGLIN So ein Fund verschlingt schon viel Zeit und Geld, allein die Bergungs- und Transportkosten, aber auch das zusätzliche Personal, das sich um die Freilegung kümmert. Drei bis vier Personen sind ständig mit Ausgraben und Dokumentieren beschäftigt, zudem unterstützen uns zwei Restauratorinnen vom Historischen Museum. Man darf auch nicht den höheren Aufwand für Öffentlichkeitsarbeit 
vergessen, den ein solcher Fund mit sich bringt. Es kommen viele Kollegen und Kolleginnen sowie Professoren und Professorinnen mit ihren Studierenden aus dem In- und Ausland. Das grosse Interesse an der Deponierung ist toll: Es gibt uns die Chance, den Fund in den Kontext der gesamten Siedlung und der zugehörigen Gräberfelder zu stellen.

\section{Habt ihr die Einzigartigkeit des Depots denn gleich erkannt?}

S. HÜGLIN Nein, zu Beginn hat man ja nur einen Buntmetallring im Profil gesehen, den haben wir erst gar nicht als latènezeitlich erkannt. Ich dachte zuerst, es sei vielleicht der Henkel von einem Farbeimer aus der Chemie.

N. SPICHTIG Der Ring wurde in einem Leitungskanal, also in einer modernen Störung sichtbar. Es hätten genauso gut die Reste einer Leitung sein können. Erst als wir die darüber liegenden Schichten abgebaut haben, wurde klar, dass es sich um eine Deponierung handelt.

S. HÜGLIN Wir haben dann sofort spezielle Dokumentationsmethoden eingesetzt. Jede Schicht wurde nicht nur fotografiert, gezeichnet und beschrieben, sondern zusätzlich noch dreidimensional gescannt. Als unter der Keramik weitere Metallgegenstände zu Tage kamen, wussten wir, dass es ein ganz aussergewöhnlicher und wertvoller Fund ist, also dass wir auf eine Art «Schatz» gestossen sind.

N. SPICHTIG So viele qualitätvolle bemalte ganze Gefässe sind sehr selten. Auf dem gesamten Siedlungsareal haben wir bislang über 500 Gruben freigelegt, die z.B. als Vorratsspeicher, Getreidesilos oder Werkplätze angelegt worden waren; aber die meisten Gefässe, die wir bisher gefunden haben, sind zerschlagen, da es sich im Wesentlichen um Abfall handelt. Allein die grosse Zahl ganzer Keramikgefässe ist schon aussergewöhnlich. Wir können sogar weiter gehen: Auch ausserhalb Basels gibt es keine genau vergleichbaren Fundensembles. Ansatzweise könnte man aus der Schweiz die Funde vom «Wauwiler Moos» im Kanton Luzern vergleichen, wo schon Ende des 19. und Anfang des 20. Jahrhunderts beim Torfstechen neben Seitenteilen eines Feuerbocks einige Kesselbestandteile und Metall- 
geräte in einem Depot gefunden wurden. Auch die Niederlegung von einzelnen Keramikgefässen im Moor ist belegt, aber eben nicht in einem einzigen, so reichhaltigen Depot. Es könnte zwar sein, dass unser Bild verzerrt ist, weil bei Depotfunden Anfang des letzten und im vorletzten Jahrhundert die Keramik möglicherweise nicht immer aufgehoben wurde und nur die Metallgegenstände gesammelt wurden, aber trotzdem bleibt die Basler Deponierung bisher einzigartig.

S. HÜGLIN Es gibt in der Normandie, im Seinetal westlich von Rouen eine Deponierung mit einer Brandbestattung in einer zylindrischen Grube (Tombeau de la Grande-Houssaye, Mailleraye-sur-Seine, Dép. Seine-Maritime). Von der Art der Niederlegung, der Zahl und Art der Beigaben ist dieser Fund unserem Depot - von dem wir noch nicht wissen, ob es ein Grab sein könnte - in mancher Hinsicht vergleichbar. Allerdings gibt es dort zusätzlich noch Teile eines Wagens. Im British Museum werden derzeit die so genannten Chiseldon Cauldrons untersucht. Das ist eine Deponierung aus der Nähe von Swindon mit mindestens 12 Kesseln, die unseren von der Machart her sehr ähnlich sind. Die Hälfte der Kessel lag mit der Öffnung nach unten, die restlichen auf der Seite zusammen mit zwei Kuhschädeln in einer Grube. Aber damit enden die Vergleiche schon. Das macht eine Interpretation so schwierig. Ausserdem erkennen wir manche Funde erst beim Röntgen.

\section{Wie bitte?}

S. HÜGLIN Ja, manche Objekte sind so zerbrechlich oder korrodiert, dass wir sie nur teilweise freilegen können und sie stattdessen lieber als handliche Blöcke bergen, um sie im Unispital mit dem Computertomographen untersuchen zu lassen. Da kann man dann die genaue Form der Gegenstände und die Zusammenhänge viel besser erkennen. Z.B. haben wir lange gedacht, wir hätten eine Lanzenspitze mit verbogenem Schaft gefunden. Doch die CT-Aufnahmen haben uns eines Besseren belehrt: Es sind wahrscheinlich Teile einer Schere; in keltischer Zeit sahen die aus wie solche, die man heute noch zur Schafschur benutzt. 
Als die Bedeutung des Befundes klar wurde, wie konntet ihr inn in der gegebenen Zeit retten?

S. HÜGLIN Kurz nach der Entdeckung - Norbert war gerade im Urlaub - gab es immer wieder heftige Unwetter. Einmal ist dabei eine Holzlatte vom Zeltdach auf den Fund heruntergefallen. So konnte es nicht weitergehen, es brauchte einen Grundsatzentscheid. Die Funde waren viel zu fragil, als dass man sie schnell hätte ausgraben können. Normalerweise schaffen wir an einem Tag - je nach Erhaltung ungefähr eine Schicht in einer Grube; hier brauchten wir eine Woche, wenn nicht noch länger. Unter den Fragmenten einer Keramikschale kamen Teile des grossen Metallkessels zum Vorschein, aber es sah weniger aus wie Blech, sondern wie grüner Blätterteig. Ein Windhauch und es hätte alles verblasen! Wir haben ihn schnell wieder abgedeckt. Es war ein Alptraum.

N. SPICHTIG Man muss dazu sagen, dass das etwa $50 \mathrm{~m}$ hohe Silogebäude, neben dem der Fund lag, eine wichtige Funktion beim Rückbau des Geländes hatte und ein Abbruch des Gebäudes parallel zur Grabung viel zu gefährlich gewesen wäre. Wir mussten also eine Entscheidung treffen. Ins Auge gefasst wurde zunächst, Nachtschichten zu machen oder alles wieder zuzuschütten und nach dem Rückbau des Gebäudes zu untersuchen. Als dritte Möglichkeit stand die Blockbergung zur Diskussion.

S. HÜGLIN Wir hatten auch Bedenken der Sicherheit wegen. Was wäre gewesen, wenn jemand nachts ins Areal eingedrungen wäre und wie eine Wildsau darin rumgewühlt hätte, um an die wertvollen Gegenstände zu kommen, und uns den Befund zerstört hätte? Dann wäre die ganze Mühe umsonst gewesen! Deshalb haben wir uns für eine Blockbergung entschieden.

N. SPICHTIG Ausschlaggebend war auch, dass die Bedingungen für eine sachgerechte Ausgrabung eines so komplexen Fundes in einem Gebäude viel besser sind. 
Abb. 4 Hohlräume werden mit Glasblähkügelchen ausgefüllt, damit sie nicht in sich zusammensacken. Glasblähkügelchen sind leichter und weniger spitz als Sandkörner. Ausserdem unterscheiden sie sich farblich gut vom Befund und lassen sich später gezielt mit dem Staubsauger entfernen. Foto: Michael Wenk.

Abb. 5 Nachdem die Hohlräume verfüllt und die Metallobjekte zugedeckt sind, wird Polyethylen-Granulat aufgefüllt. Es soll verhindern, dass die Objekte beim Transport verrutschen und kann später leicht wieder abgesaugt werden. Foto: Michael Wenk.

Abb. 6 Um den freistehenden, innen teilweise hohlen Block zu stabilisieren und Unebenheiten auszugleichen, wird er mit einer dicken Schicht Gips ummantelt. Der Maschendraht dient dabei als Armierung. Foto: Ilona Troxler.

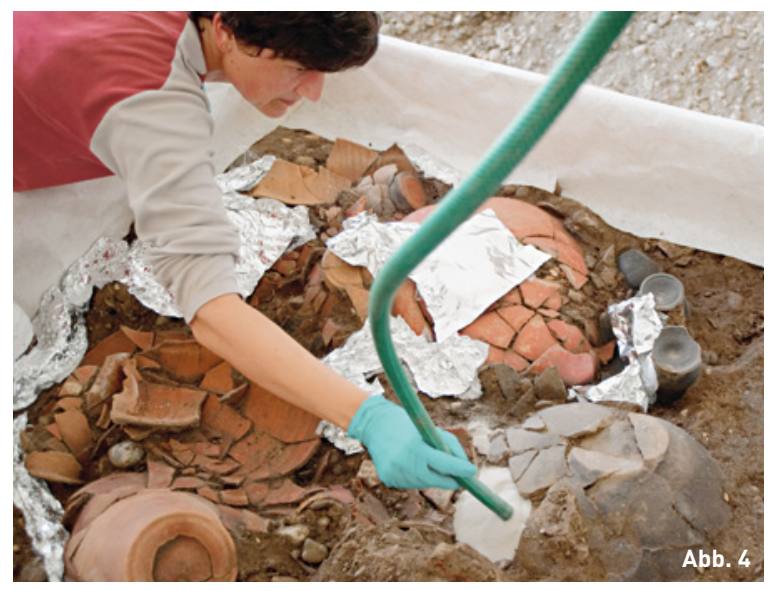

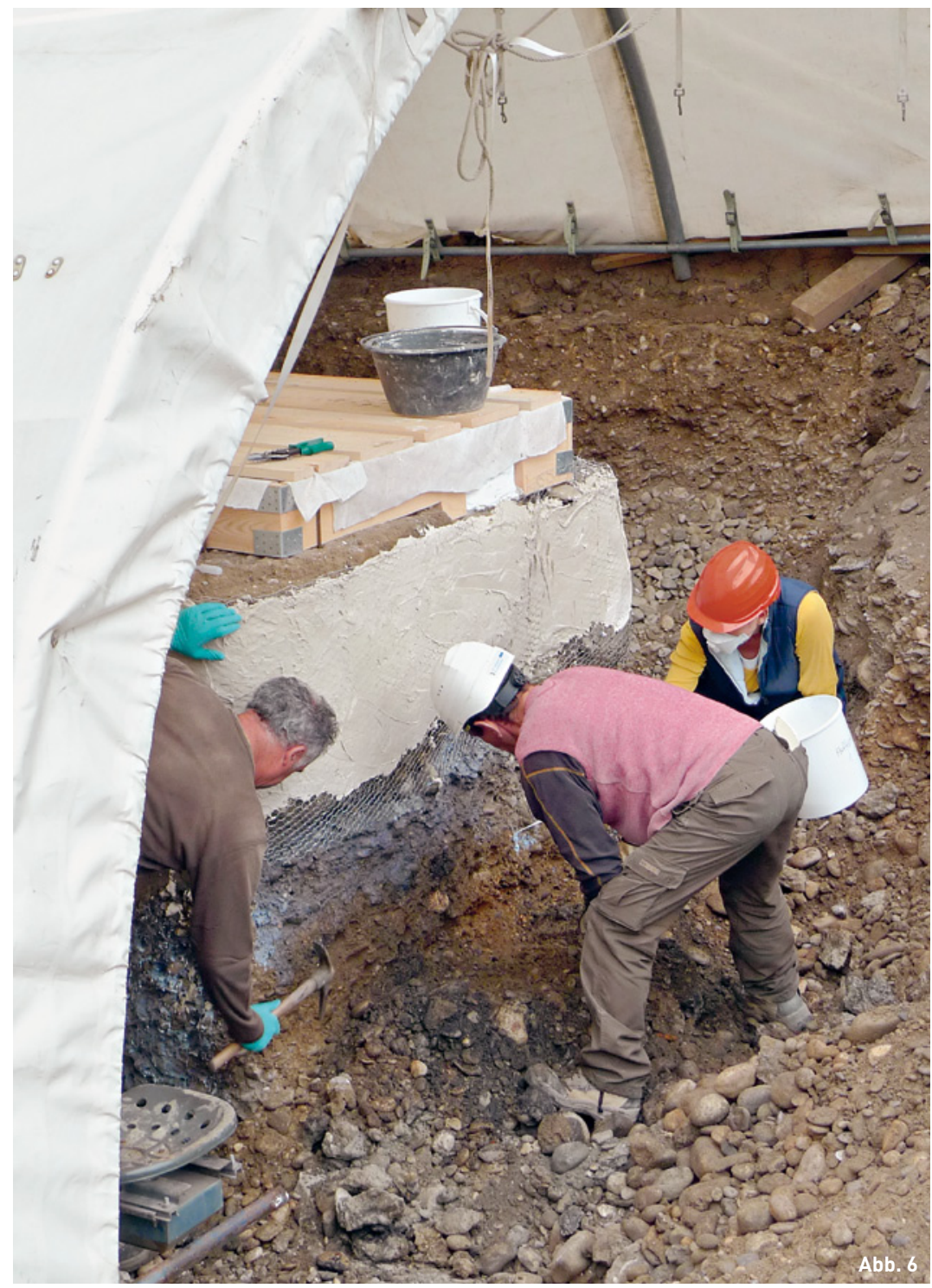

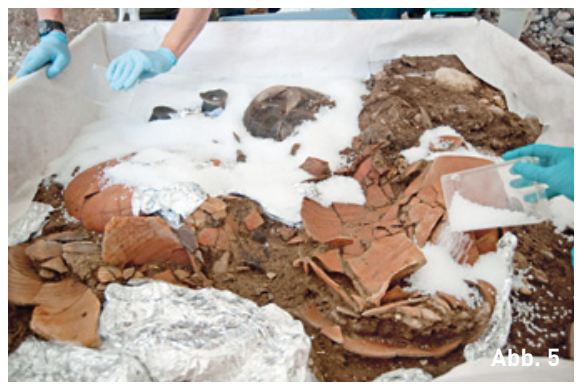




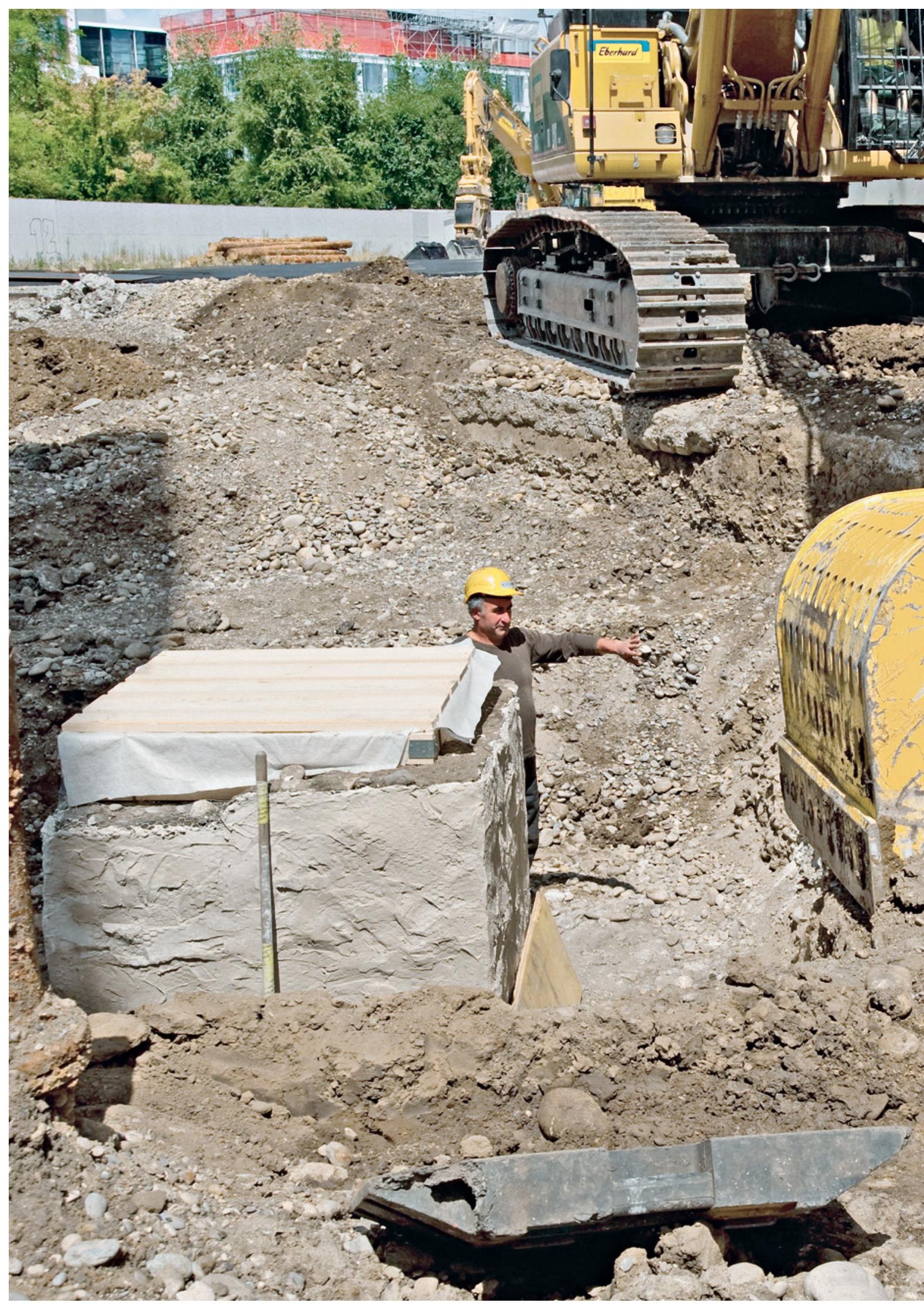


Abb. 7 Von der Kelle zum Schwer-

gerät: mit einem 50t-Bagger wird der

Abraum um den Block entfernt, um

Platz für die weiteren Vorbereitungs-

arbeiten zu schaffen. Foto: Michael

Wenk.

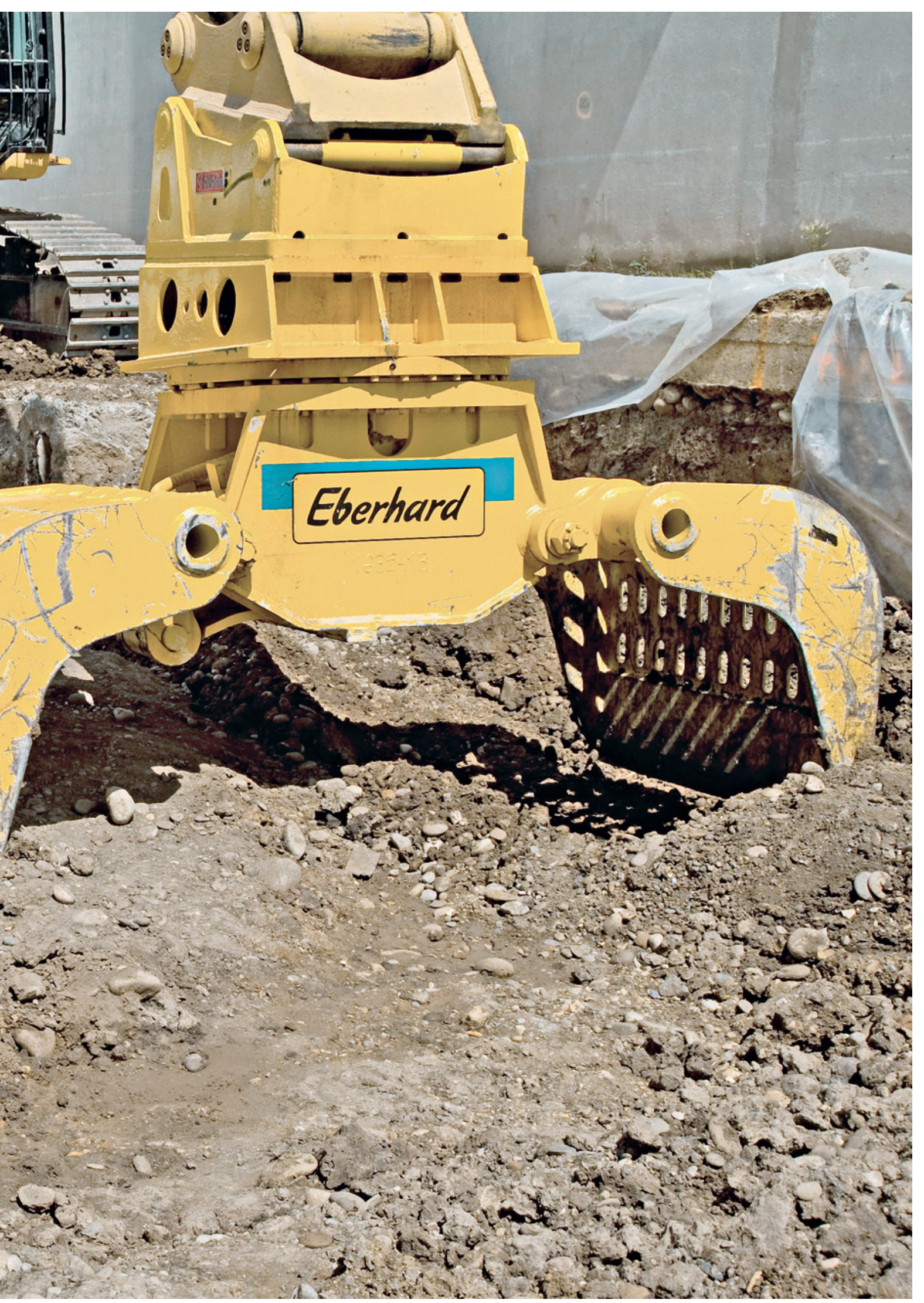


War die Blockbergung aber nicht auch ein enormes Risiko? Man kannte die Tiefe der Depotgrube doch gar nicht.

S. HÜGLIN Mit einem Metallsuchgerät habe ich geprüft, bis in welche Tiefe mit Metallgegenständen zu rechnen ist. Eine Restunsicherheit und deshalb auch Anspannung blieb da schon. Es hätten ja noch eine Bestattung oder andere Gegenstände, die nicht aus Metall sind, tiefer liegen können.

N. SPICHTIG Erschwerend kam hinzu, dass wegen des natürlichen Rheinkieses unter dem Depot eine Blockbergung nach herkömmlicher Weise kaum durchführbar gewesen wäre. Normalerweise verschalt man den Block zuerst und schiebt dann eine Platte darunter, um den Block abzulösen. Hier im Kies wäre das unmöglich gewesen. Deshalb haben Spezialisten eine aufwendige Konstruktion machen müssen. Einige Zentimeter unter dem Niveau, auf dem wir das Ende des Depots vermuteten, wurden in zwei Lagen Bohrungen durchgeführt, die um 90 Grad zueinander versetzt waren. So entstand ein Gitternetz aus hohlen Bohrstäben. Zusätzlich wurde in diese Rohre noch Kunstharz eingespritzt, das die Zwischenräume abdichtete. Während der Bohrungen haben wir ganz genau darauf geachtet, ob das Material, das rauskommt, nicht doch irgendwelche Funde enthält. Wir hätten dann sofort gestoppt.

Am Ende wog der Block fast 9 Tonnen. Gab es keine statischen Probleme?

S. HÜGLIN Die Bauleute kennen sich mit so schweren Lasten gut aus, und wo sie selbst nicht mehr weiterwussten, haben sie Spezialisten engagiert. Mit einem Kran wurde der Block von der tiefer gelegenen Grabung auf die Strasse gehievt und dann mit einem Schwerlast-Gabelstapler angehoben und in die Ladeluke des Gebäudes hinein gesetzt. Im Gebäude selbst war zur Sicherheit der Keller mit Baumstämmen ausgespriesst worden. Wäre der Block dort nur abgesetzt worden, wäre es kein Problem gewesen, aber der Statiker hatte Bedenken, weil der Block auf kleinen Rollen quer durch den ganzen Raum bewegt werden musste. 
N. SPICHTIG Die grösste Herausforderung war eigentlich der Block selbst! Wir befürchteten, dass etwas aus dem Gitterboden rausbricht, aber es lief alles problemlos. Es war absolut perfekt von den Bauleuten und den Spezialisten durchdacht und ausgeführt.

Unter Laborbedingungen wird der riesige Block in unglaublicher Feinarbeit freigelegt. Könnt ihr schon jetzt etwas über die Funde sagen?

S. HÜGLIN Es sind zunächst einmal unheimlich viele Objekte, oft in vielen Einzelteilen. Ich habe den Eindruck, dass hier die wertvollsten Gegenstände aus dem Besitz eines sehr vermögenden Mannes sehr sorgfältig aufeinander gestapelt und vergraben worden sind.

N. SPICHTIG Die bemalte Keramik, die Kessel, aber auch der hölzerne Eimer mit den Metallbändern und das Pferdegeschirr, all das weist auf eine gehobene Stellung der einstigen Besitzer hin. Vieles, wie die Form und die Qualität der Gefässe, liesse sich als Zubehör eines Banketts interpretieren. Andererseits gehören das Pferdegeschirr und der mutmassliche Halsring zur persönlichen Habe. Bislang ist jedoch noch nicht alles ausgegraben, und mit dem Halbwissen, das wir momentan haben, bleibt es eine Hypothese, ob die Objekte von einer Person oder einer Gemeinschaft stammen. Die aus Buntmetall gegossene Pfanne ist sicher ein Import. Auch der Mörser gehört eher nicht zu den keltischen Alltagsküchenutensilien.

S. HÜGLIN Ja, der Mörser, darin hat man Kräuter und Gewürze zerrieben. Dann noch die importierte Pfanne aus gegossener Bronze und die Trinkgefässe für den Wein, der nachweislich aus der Toskana bezogen wurde - das alles weist darauf hin, dass die Kelten hier viel aus der mediterranen Küche, ja vielleicht auch andere Elemente des Lebensstils aus dem Süden übernommen haben. 


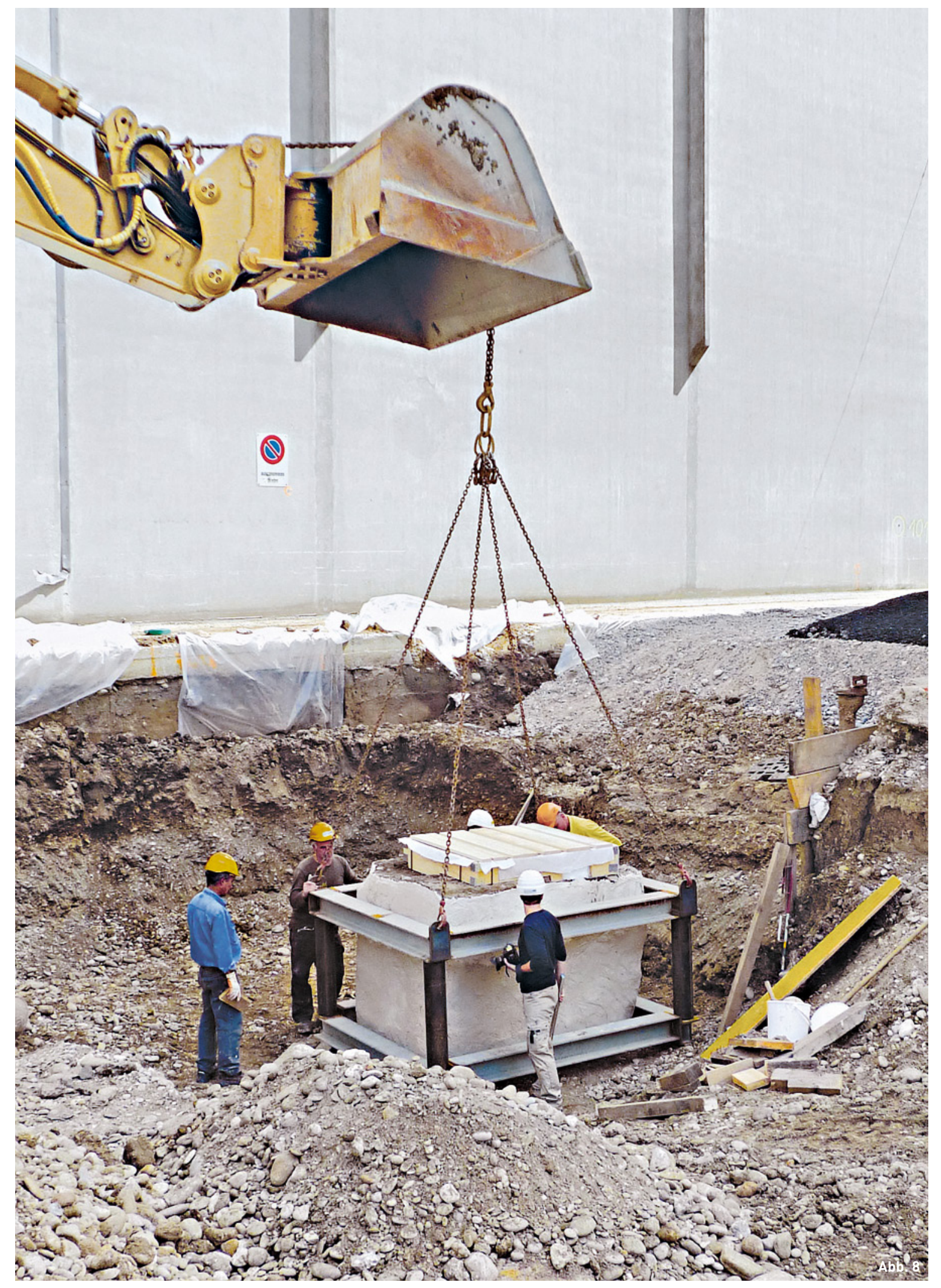


Abb. 8 Der massgefertigte Stahlrahmen wird mit Hilfe eines Baggers von oben über den Block gesetzt. Eine Holzverkleidung zwischen Erdblock und Stahlrahmen soll den Jahrhundertfund für den Transport schützen. Die Lücke zwischen den Bohlen und dem Block wird zudem noch mit Sand verfüllt. Foto: Michael Wenk.

Abb. 9 Präzisionsarbeit: Die hydraulische Bohrung für das Bodengitter muss von Spezialisten durchgeführt werden. Doch auch für sie ist die Situation neu, denn zum ersten Mal bohren sie unter einem archäologischen «Schatz» hindurch. Foto: Denise Grossenbacher.

Abb. 10 Nachdem die Hohlräume um die Stahlrohre und Armierungseisen aussen mit PU-Schaum abgedichtet wurden, füllen Spezialisten die Rohre nun mit einer besonderen Epoxidharzmischung auf. So soll ein stabiler Boden entstehen. Foto: Norbert Spichtig.
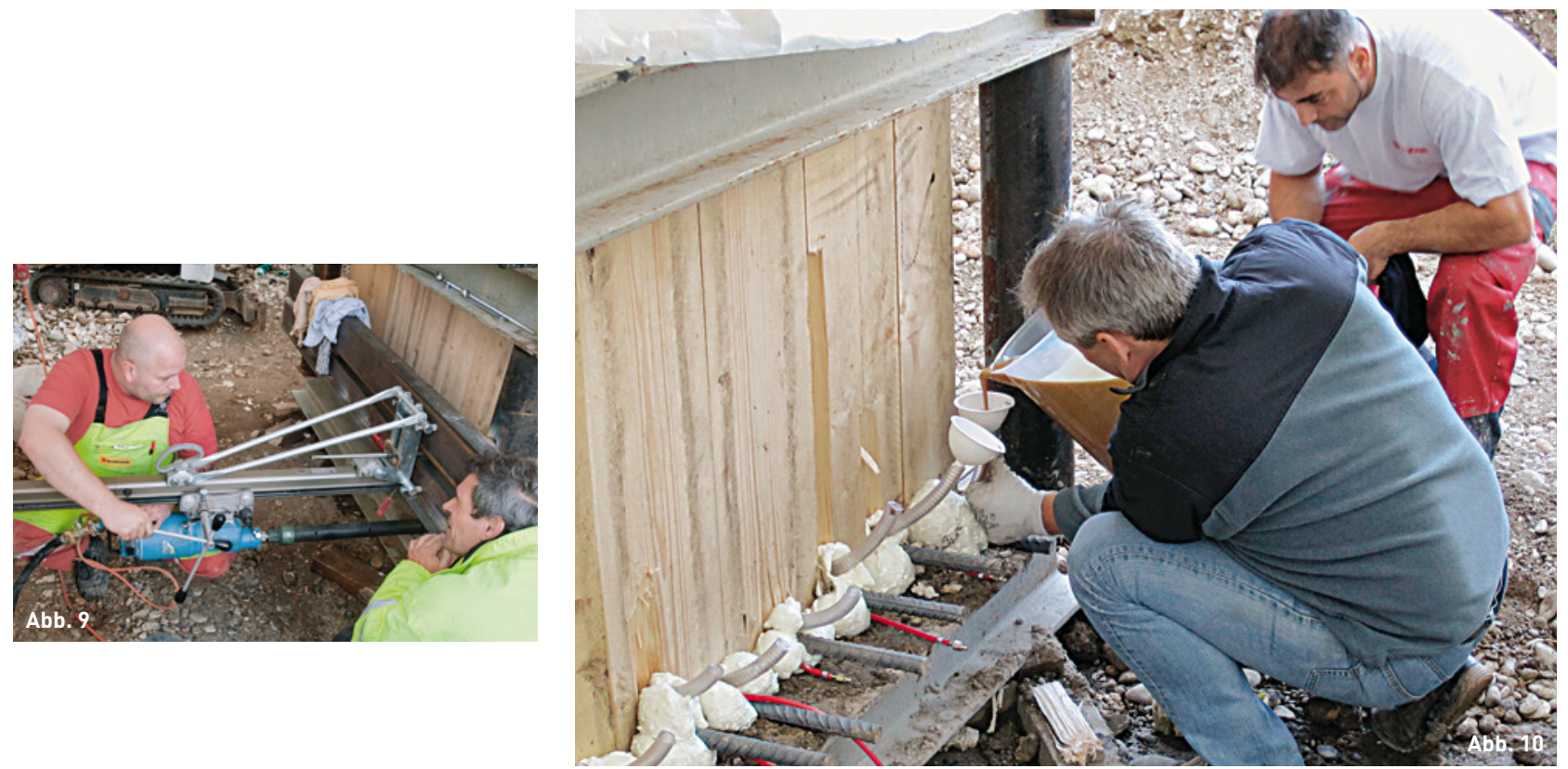


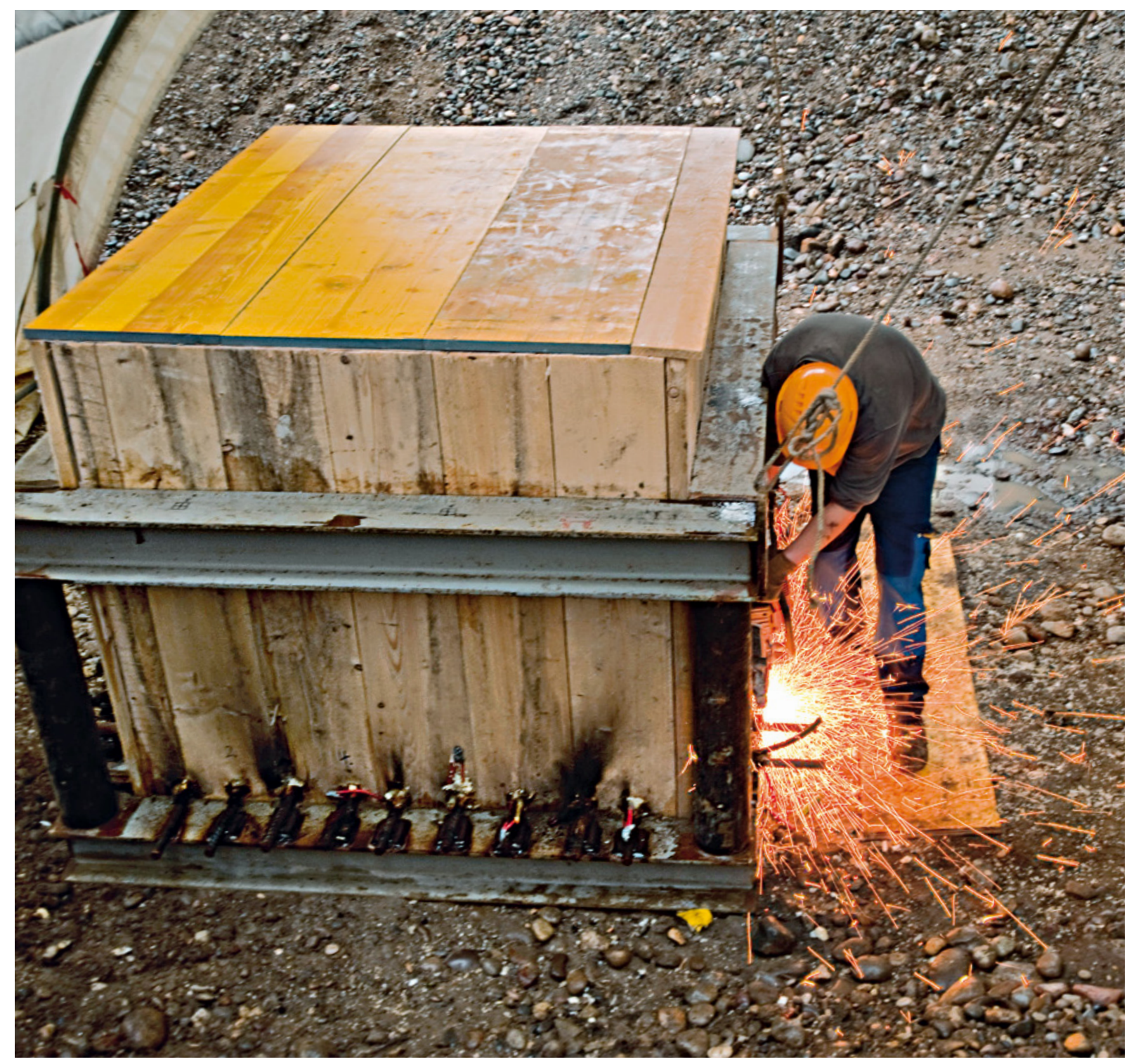


Benutzten sie eventuell auch mediterrane Gewürze?

N. SPICHTIG Das wissen wir nicht; Hinweise darauf fehlen. Aber z.B. anhand von abgebauten Fetten an der Keramik lässt sich schon einiges über die Verwendung der Gefässe feststellen. Solche Aspekte sind natürlich in die Auswertungen mit einzubeziehen.

S. HÜGLIN In einem kleinen Töpfchen - bisher übrigens das einzige handgemachte Gefäss, das wir gefunden haben befanden sich Reste einer klebrigen dunklen Masse. Vermutlich handelt es sich aber nicht um eine Speise, sondern um eine Art Teer, also ein Schmier-, Klebe- oder Dichtungsmittel. Wahrscheinlich ist dieser Topf nur wegen seines Inhalts ins Depot gelangt. Alle anderen Gefässe, die wir bisher entnommen haben, sind umgekehrt niedergelegt worden und waren leer.

Warum könnte diese Deponierung angelegt worden sein, was gibt es da für Hinweise?

N. SPICHTIG Offenbar ging es nicht um einen Inhalt, wenn die Gefässe so eng ineinander gestapelt und auf den Kopf gestellt worden waren. Vielmehr scheint das strukturierte Niederlegen der Objekte selbst im Vordergrund gestanden zu haben, wie man es vor allem aus religiösen Kontexten kennt.

S. HÜGLIN Ich habe mir überlegt, warum die Gegenstände so kreisförmig angeordnet sind: Ich denke, dass sie in einem Holzfass vergraben wurden. Das Holz ist natürlich nach so langer Zeit fast vollständig vergangen. Dann hat sich die Verfüllung der Grube gesetzt, in der das Fass stand, so dass der Inhalt nicht nur zusammengedrückt, sondern auch seitlich verschoben wurde. Der Durchmesser des Fasses dürfte einen Meter und die ursprüngliche Höhe wohl etwa zwei Meter betragen haben. Das kommt ungefähr den Massen späterer römischer Fässer gleich, z.B. jenen aus Eschenz im Thurgau. Ein Meter entspricht übrigens ziemlich genau drei keltischen «Fuss». 
N. SPICHTIG Es gibt aber bis anhin keinerlei Spuren von einem Fass. Zwar erwähnt schon Caesar in seinen Beschreibungen Fässer, und die frühesten Nachweise kennen wir aus dem späten 1. Jahrhundert v. Chr. aus der keltischen Siedlung von Manching in Bayern; dennoch möchte ich mich nicht auf solche Spekulationen festlegen. Beim derzeitigen Stand gibt es keine Belege für ein Fass.

Ich halte ein Behältnis nicht für ausgeschlossen, aber es wäre z.B. auch denkbar, dass die Objekte in Stoff oder Leder gehüllt waren oder nur so in einer kleinen runden Grube deponiert wurden. Es müssen einfach auch andere Möglichkeiten in Betracht gezogen werden.

S. HÜGLIN Deswegen ist es uns so wichtig, jedes Steinchen, jeden Knochen und jede noch so kleine Störung genau zu dokumentieren, damit wir klären können: Was gehörte ursprünglich zum Depot und was ist später reingerieselt? Teilweise gab es grosse Hohlräume innerhalb oder unterhalb der Gefässe. Manche Hohlräume waren aber auch zum Teil mit lockerem Material verfüllt. Das hat mich stark an einen Ameisenhaufen erinnert. Einmal haben wir darin ganz viele kleine Schnecken gefunden. Die Aktivität von Tieren unter der Erde sollte man nicht unterschätzen.

N. SPICHTIG Aber die Herkunft mancher Steine kann man weder mit Schnecken noch mit Ameisen einfach erklären, wenn man von einem einst geschlossenen Raum ausgeht. Die Situation ist sehr kompliziert. Es sind zwei Gruben, die sich überlagern, und darin wurden später die Objekte deponiert. Wir müssen nun herausfinden, wie es mit der Verfüllung darum herum aussieht. Warum sollte die Kreisform des hypothetischen Fasses erhalten geblieben sein? Wäre der Druck von oben gekommen, hätte sich der Kreis grosso modo erhalten, aber dann liesse sich die beobachtete Lage der Objekte kaum mit einem Behältnis in Einklang bringen, das dem Druck nachgegeben hat. Hätten die Kräfte schräg von der Seite eingewirkt, müssten wir bei einem verrottenden Holzbehälter - von oben gesehen etwa eine elipsoide Form finden. Wir sollten auch die Ergebnisse der Mikromorphologie abwarten. 
S. HÜGLIN Auf jeden Fall müssen wir offen bleiben für alle Beobachtungen. Das dreidimensionale Modell wird uns helfen, die späteren Setzungsprozesse zu verstehen und erst dann können wir auch weiter darüber nachdenken, wie die Objekte ursprünglich deponiert wurden.

\section{$\underline{\text { Für dieses Modell benutzt ihr eine besondere Software... }}$}

N. SPICHTIG Ja. Anfangs haben wir jede Lage mit einem 3DScanner abgetastet, damit wir ein digitales Oberflächenrelief bekamen. Das war sehr aufwendig, allein das Scannen hat viel Zeit in Anspruch genommen. Jetzt benutzen wir ein Verfahren, das aus vielen Fotos, die man von allen Seiten des Objekts aufnimmt, ein 3D-Modell erstellt.

S. HÜGLIN Das spart uns sehr viel Zeit, auch wenn der Computer manchmal für ein 3D-Modell die ganze Nacht rechnen muss.

\section{$\underline{\text { Habt ihr eine Idee, warum das Depot niedergelegt wurde? }}$}

S. HÜGLIN Das ist eine sehr schwierige Frage. Dafür sollten wir abwarten, bis wir alles freigelegt haben. Wir können nur Weiteres herausfinden, wenn wir die Schichten um die Deponierung herum und die Strukturen in der Umgebung des Fundes genau untersuchen. Ich habe beobachtet, dass über den beiden Gruben und dem Depot eine Kiesschicht liegt, welche die Bewohner von damals wohl bewusst aufgebracht haben, um eine trockene und saubere Oberfläche zu erhalten. Ganz in der Nähe der Grube haben wir Schwellbalkengräbchen, also Spuren von Holzgebäuden gefunden, zu denen die Gruben gehört haben könnten. Reste einer oberirdischen Markierung des Depots konnten wir nicht finden. Ich denke deshalb, dass das Fass samt seinem Inhalt versteckt wurde. Vielleicht ist es aber auch ein Grab? Womöglich finden wir tatsächlich noch eine Brandbestattung. 
N. SPICHTIG Bislang haben wir aus der Gasfabrik keine Hinweise auf Kremationen ...

S. HÜGLIN ... aber für ein Skelett hat es eigentlich keinen Platz, höchstens für einen Schädel als pars pro toto. Ausserdem sahen die Befunde mit menschlichen Skelettresten bisher immer ganz anders aus. Auf jeden Fall wollte man offenbar vermeiden, dass jemand die wertvollen Stücke findet. Stratigrafisch sind aus meiner Sicht die Deponierung und die Kieslage eher an das Ende der Besiedlungszeit, also etwa zwischen 100 und 80 v. Chr. zu datieren.

N. SPICHTIG Für eine zeitliche Einordnung des Depots sollten primär die Funde selbst, aber natürlich auch die Objekte aus stratigrafisch älteren und jüngeren Schichten bzw. Strukturen beigezogen werden.

Wenn das Depot so wertvolle Gegenstände enthält: Gibt es auch sonst in der Siedlung Hinweise auf Reichtum oder höher gestellte Personen?

S. HÜGLIN Einerseits bestehen gravierende Unterschiede zwischen den ländlichen Siedlungen in der Umgebung und der Grosssiedlung hier. Während zum Beispiel bestimmte Funde wie Amphoren auf dem Land selten sind, haben wir grosse Mengen davon. Andererseits gibt es aber auch innerhalb der Siedlung gewisse Unterschiede. Es gibt aber nicht nur eine, sondern mehrere Siedlungsparzellen, auf denen sich Importe und andere kostbare Gegenstände häufen. Das spricht dafür, dass es eine Reihe von Angehörigen der gesellschaftlichen Elite an verschiedenen Orten innerhalb der Siedlung gab. Bei den Bestattungen konnten wir inzwischen feststellen, dass es sich bei den Skeletten innerhalb der Siedlung wohl um eine spezielle Bestattungssitte handelt und nicht um Knochen, die mit dem Siedlungsabfall in Gruben entsorgt wurden. Im Schnitt haben diese Bestattungen sogar mehr und wertvollere Beigaben als die Toten auf dem Gräberfeld. 
Ist man früher nicht davon ausgegangen, dass es sich bei den Skelettresten in den Gruben um Ausgestossene oder um Opfer feindlicher Überfälle handelte?

S. HÜGLIN Ja ganz richtig. Heute denken wir eher, dass es sich um Bestattungen handelt, und dass es sogar ein Privileg von Angehörigen der Elite war, innerhalb der Siedlung vielleicht in der Parzelle, in der sie gewohnt hatten - bestattet zu werden. Möglicherweise wurden so herausragende Personen in den Kreis der Ahnen aufgenommen. Es gibt vieles, was wir aus heutiger Sicht als gruselig empfinden, aber in anderen Kulturen ist es zum Beispiel die grösste Ehrerbietung, wenn der Schädel vom Opa im Regal steht. Christliche Reliquien sind ja auch Skelettteile von Menschen, die ganz besonders verehrt werden.

N. SPICHTIG Der komplexe Umgang mit den Toten in BaselGasfabrik ist Gegenstand eines spannenden Auswertungsprojektes zahlreicher Disziplinen, das 2011 begonnen wurde. Was höher gestellte Personen betrifft, möchte ich gerne noch einen Fund erwähnen: den so genannten «Goldschatz von St. Louis». Ob er tatsächlich von St. Louis stammt oder vom Kegelriss bei Ehrenstetten in der Nähe von Freiburg im Breisgau, lässt sich heute nicht mehr eindeutig klären. Aber wenn er aus dem Umfeld von Basel-Gasfabrik stammen würde, würde das sehr gut in den Kontext des jetzigen Fundes passen. In diesem Schatz gibt es mehrere Goldringe und zahlreiche keltische Goldmünzen, die nicht aus der Region stammen, sondern auf Beziehungen in den Osten, nach Bayern und Böhmen, und nach Nordfrankreich weisen. Weitläufige Verbindungen also, die wohl vornehmlich über die Elite liefen. 


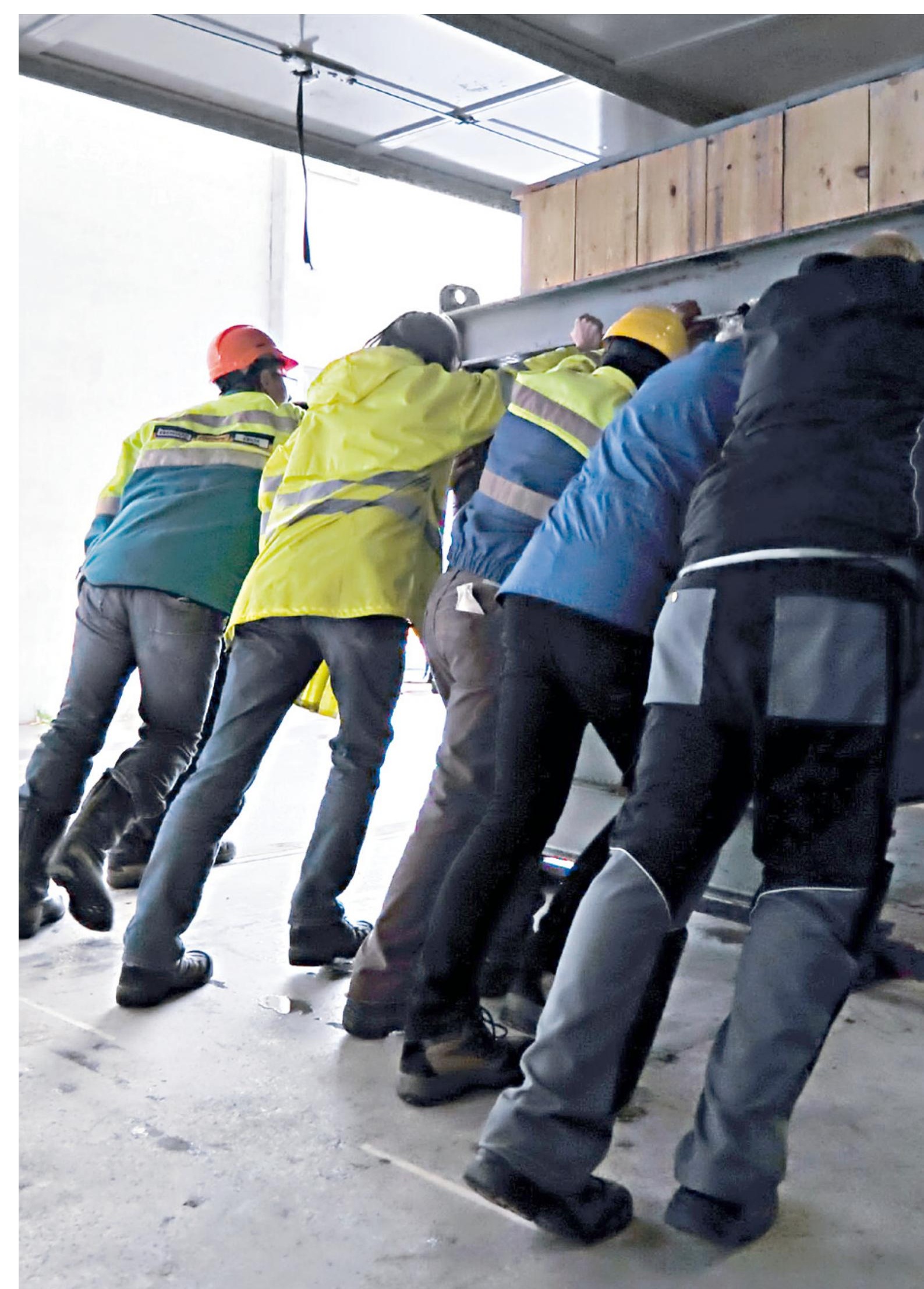


Abb. 12 Manpower pur: Auf Schwer-

lastrollen wird der Block an seinen

Platz geschoben. Damit der Geschoss-

boden standhält, wurde der darun-

terliegende Luftschutzkeller mit

Baumstämmen ausgespriesst. Foto:

Denise Grossenbacher.

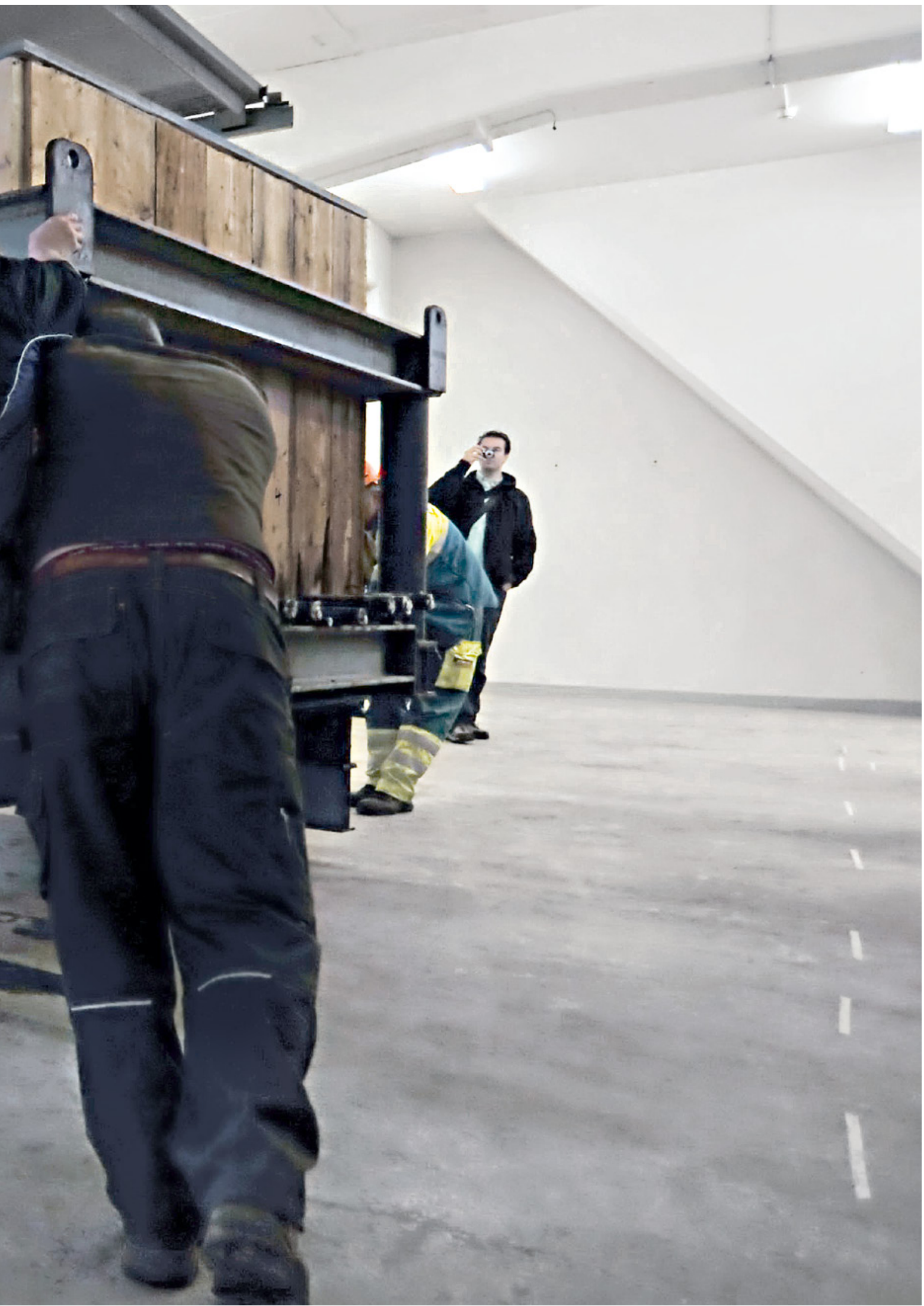


Wie geht dieser Reichtum mit der enorm hohen Kindersterblichkeit zusammen? Über die Hälfte der Toten auf den Gräberfeldern sind Kinder - wie lässt sich das erklären?

N. SPICHTIG Es ist das Normale, was in einer traditionellen Bevölkerung zu erwarten ist; früher hat man die kleinen Knochen nicht entdeckt. Das haben die Anthropologen immer beklagt. Heute finden wir aufgrund der verfeinerten Grabungsmethoden viel eher die winzigen Knochen, selbst von Neugeborenen oder Föten. Das war zu Stehlins Zeit noch nicht gegeben.

Das Leben am Rhein verhalf den Bewohnern von Basel-Gasfabrik also offenbar zu Wohlstand. Warum aber liessen sie sich so nah am Rhein nieder, wo die Gefahr von Überschwemmungen und damit Krankheiten, bedingt durch Feuchtigkeit und Sümpfe gross ist?

N. SPICHTIG Im Unterschied zur keltischen Siedlung im Klybeck auf der gegenüberliegenden Rheinseite war hier kein feuchter Untergrund. Die Siedlung liegt über dem Hochwasserniveau. Das Wasser war kein Problem, vielmehr ist es der Boden. Seine Qualität ist für Landwirtschaft nicht ideal.

S. HÜGLIN Den Bewohnern ging es in erster Linie sicher nicht um Landwirtschaft, sondern um den Hafen und den Rheinübergang - also um Handels- und Austauschverbindungen. Wir haben kaum landwirtschaftliche Geräte in der Siedlung gefunden, dafür aber technische Einrichtungen wie Töpferöfen und Schmieden. Trotzdem wurde in manchen Gruben viel Getreide gelagert. Es muss also einen regen Austausch zwischen dem ländlichen Umfeld und der Siedlung gegeben haben. 
Abb. 13 Auf den Kopf gestellt - fast alle Gefässe des Depots waren leer und mit der Öffnung nach unten ineinander gestapelt niedergelegt worden. Foto: Julia Imhoof.

Abb. 14 Mit Zahnarztbesteck, Holzstäbchen, Pinsel und Staubsaugerdüse en miniature: Behutsam befreit ein Team von Archäologen und Restauratorinnen die 2100 Jahre alten

Objekte von der umliegenden Erde. Foto: Andreas Niederhäuser.

Abb. 15 Als hätte die rotbemalte Schale auch im Alltag zum grossen Metallkessel gehört, so passgenau bedeckte sie den Kesselboden. Damit der Kessel bei der Freilegung nicht zerbricht, mussten lose Stücke mit Streifen aus Kunstgewebe und Spezialkleber gesichert werden. Foto: Julia Imhoof.
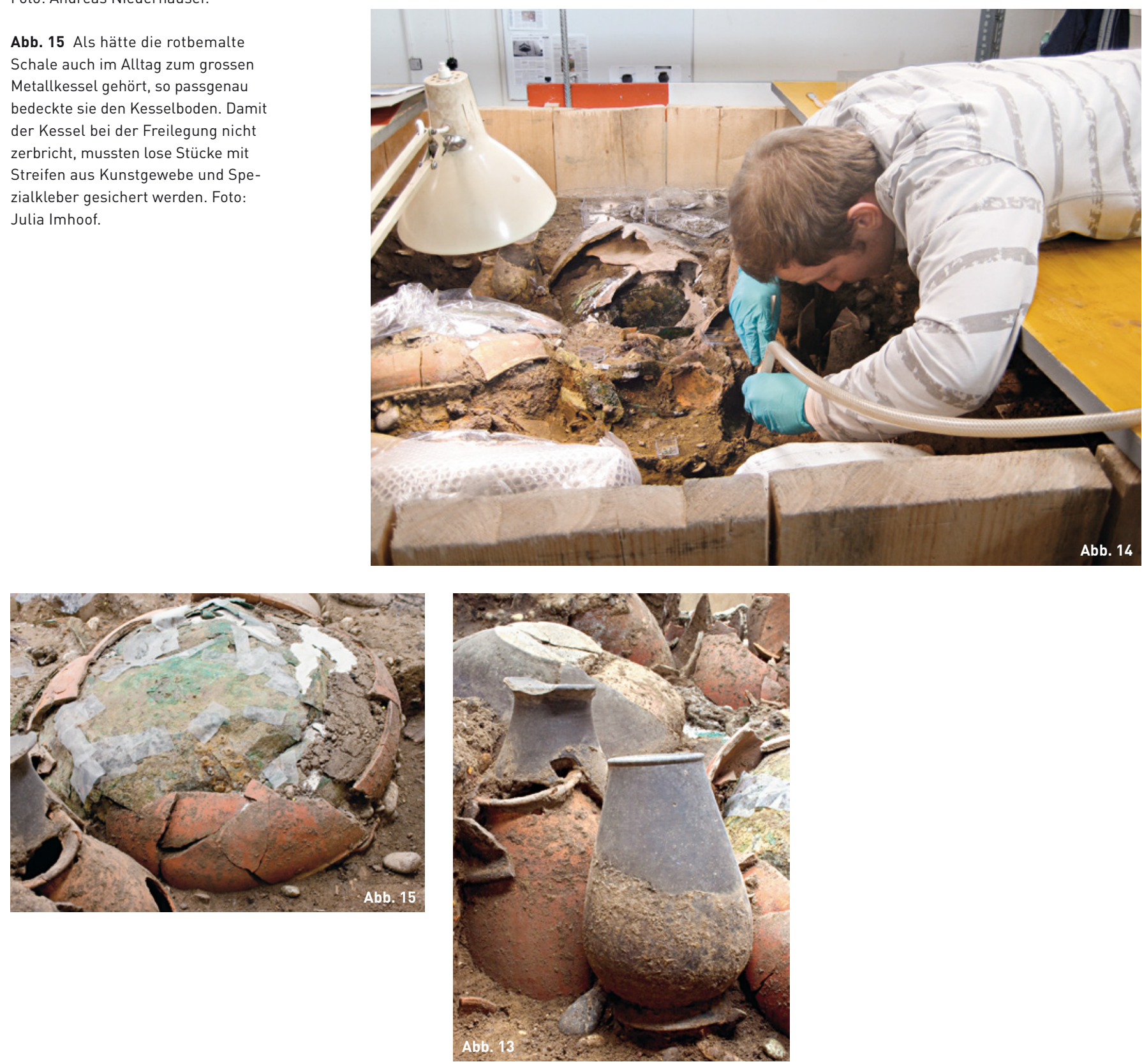
N. SPICHTIG Der Nachweis von landwirtschaftlichen Geräten ist sehr schwierig. Denn viele wurden wahrscheinlich recycelt. Das Fehlen von Ackergeräten an sich ist deshalb kein schlagkräftiges Argument. Aber in der Siedlung selbst haben wir auch keine Hinweise auf Felder.

\section{War Basel-Gasfabrik dann ein städtischer Handelsplatz?}

S. HÜGLIN Nein, wir haben bislang keine öffentlichen grossen Gebäude und keine kommunalen Einrichtungen gefunden. In einigen Parzellen konzentrieren sich zwar herausragende Funde, aber alles in allem sind die Grundstücksgrössen ähnlich. Es scheinen relativ autarke Siedlungseinheiten gewesen zu sein. Die Bebauung war locker, zwischen drin Gärten, vielleicht auch ein Gehege für kleine Tiere.

N. SPICHTIG Die Analyse der Tierknochen zeigt, dass Tiere in der Siedlung geschlachtet wurden. Die Haltung von Grossvieh in der Siedlung ist aber unwahrscheinlich. Ich gehe von einer Art Klientelsystem aus. Die Bauern aus der Umgebung versorgten die Menschen von Basel-Gasfabrik mit Nahrungsmitteln, während sie dafür Gegenleistungen wie z.B. rechtlichen oder politischen Schutz bekommen haben dürften.

Solche Grosssiedlungen entstanden Mitte des 2. Jahrhunderts v. Chr. auch an anderen Orten. Basel-Gasfabrik liegt da im Trend der damaligen Zeit. Die Komplexität und Dimension der Siedlung ist zwar anders als bei den ländlichen Siedlungen, aber die Bebauung gleicht eher einer Aneinanderreihung von vielen Gehöften. Voll entwickelte städtische Strukturen liegen noch nicht vor. Aber BaselGasfabrik bildete für das Umfeld einen zentralen Platz, wo auch Waren umgeschlagen wurden. 
Wird von all dem auch noch etwas für zukünftige Generationen erhalten bleiben? Wie sieht die Zukunft von Basel-Gasfabrik aus?

N. SPICHTIG Bis Mitte 2012 müssen wir noch $2000-3000 \mathrm{~m}^{2}$ ausgraben. Im Vergleich zu den über $100000 \mathrm{~m}^{2}$, die während der letzten 23 Jahre schon untersucht wurden, klingt das zwar nicht mehr nach allzuviel Arbeit, aber diese Fläche hat es in sich. Entscheidend ist immer die Erhaltung, und diese ist nun relativ gut. Die Arbeit geht uns sicher nicht aus - im Gegenteil!

S. HÜGLIN Nach der Grabung wird auf dem Novartis-Areal selbst nichts mehr zu sehen sein. Eine schöne Idee wäre es deshalb, wenn man zumindest virtuell noch mal durch die Siedlung gehen könnte, so wie das schon mal bei den Nordtangenten-Grabungen im Rahmen des lifeClipper2Projekts versuchsweise gemacht wurde.

N. SPICHTIG An der Rheinuferpromenade ist ein «Merkpunkt» vorgesehen, wo wir kleinere Installationen machen möchten, die auf die Siedlung hinweisen. Von der Originalsubstanz wird hier jedoch nichts erhalten bleiben, da Novartis und die kantonale Regierung ein Abkommen für die vollständige Sanierung des Areals abgeschlossen haben. Abgesehen davon gibt es kaum Befunde, die sinnvoll präsentierbar wären.

Und die Deponierung: wird man sie auch sehen können?

N. SPICHTIG Momentan stehen die Untersuchung des Depots, die Sicherung der Objekte sowie die nachfolgende Konservierung der Funde im Vordergrund. Wann es im Museum zu sehen sein wird, lässt sich deshalb noch nicht abschätzen. Aber der Fund wird sicher ein Highlight jeder Ausstellung zur keltischen Archäologie! - 


\section{DEPONIERUNG}

Toni Rey

Als «Depot» oder «Deponierung» wird in der Archäologie eine spezielle Befundgattung bezeichnet: Es handelt sich dabei um einzelne oder mehrere $a b$ sichtlich niedergelegte, vergrabene oder versenkte Objekte. Grabbeigaben, Siedlungsreste oder verlorene Gegenstände gehören nicht in diese Kategorie. Man nennt die Depotfunde auch Hort-, Versteckoder Verwahrfunde. Die Niederlegungen werden von Fall zu Fall unterschiedlich gedeutet: z. B. als Massnahme zum Schutz vor fremdem Zugriff oder als Opfergabe an die Götter. -

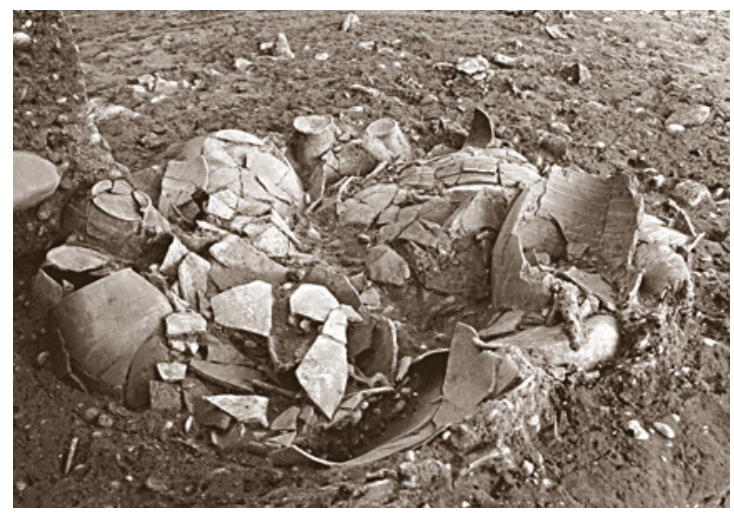

Abb. 16 Die oberste Lage der Deponierung besteht aus einer Vielzahl von Gefässen unterschiedlichster Form. Nur wenige haben dem Erddruck standgehalten.

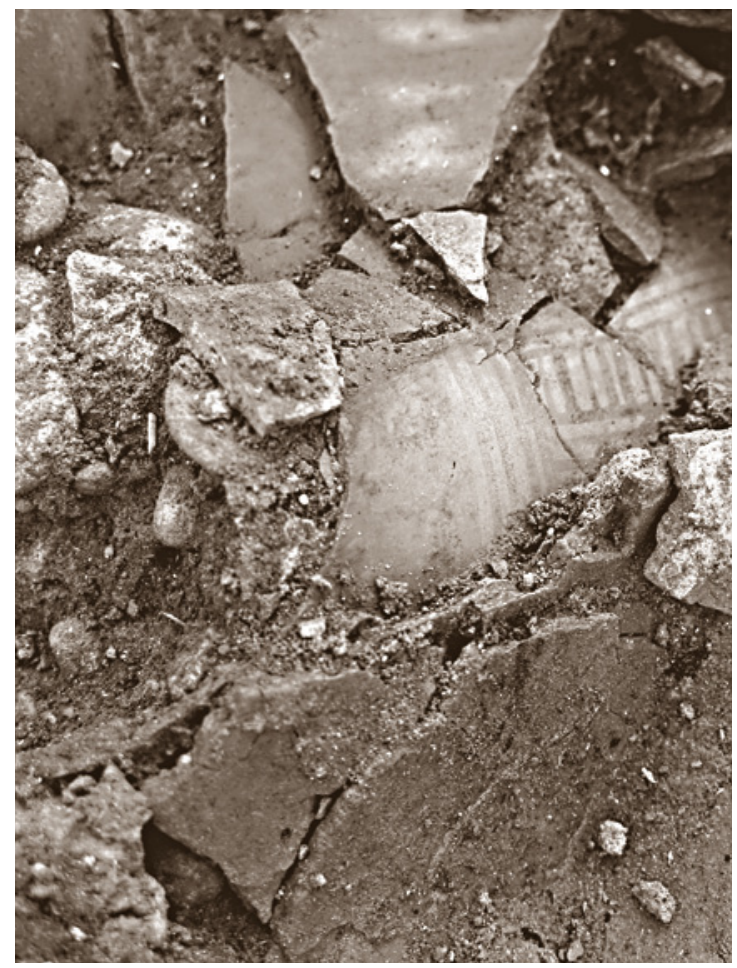

Abb. 17 Viele Keramikgefässe sind auf der Aussenseite mit geometrischen Mustern bemalt. Foto: Julia Imhoof. 


\section{VOM EINFACHEN «WOHNGRUBENDORF» ZUM REGIONALEN ZENTRUM}

Marion Benz

Die keltische Siedlung «Basel-Gasfabrik» gilt heute als die erste stadtähnliche Vorgängersiedlung $\mathrm{Ba}$ sels. Das war nicht immer so. Als im Jahr 1911 der Basler Jurist und Archäologe Karl Stehlin Spuren keltischer Besiedlung am Rheinhafen St. Johann entdeckte, wähnten sich die Basler noch als Erben der römischen Kolonie Augusta Raurica. Von der Baukunst der Rauriker hielt der Architekturliebhaber Stehlin offenbar wenig. Da sich in den Gruben, die er auf dem Bauplatz der damaligen Gasfabrik fand, Feuerstellen und andere kulturelle Hinterlassenschaften erhalten hatten, interpretierte er sie - wie damals in der Forschung allgemein üblich - als Wohngruben mit Stroh- oder Schilfdach. 1915 entdeckt er das dazugehörige Gräberfeld, und gut 20 Jahre später sind bereits mehr als 100 Gruben bekannt. Als keltisches «Wohngrubendorf» findet die Siedlung «Basel-Gasfabrik» Eingang in die Lehrbücher. Lange stört sich niemand daran, dass die Funde regen Handel von der Ostsee bis ans Mittelmeer und hochstehendes technisches Know-how belegen, während die Wohnstätten so primitiv gewesen sein sollen. Mit der fortschreitenden Erforschung durch Stehlins Nachfolger Rudolf Laur-Belart wird klar, wie die zahlreichen Pfostengruben und schmalen Gräbchen zu interpretieren sind: als Spuren oberirdischer Holzbauten. Im Sommer 1939 dokumentiert Laur-Belart erstmals «ein ganzes System sich rechtwinklig schneidender Gräbchen», die seiner Meinung nach nur von Schwellbalken stammen konnten. Er kommt zu dem Schluss, dass sich die Gruben nicht zum Wohnen eigneten.

Wenngleich Laur-Belarts Grabensysteme nach heutigem Kenntnisstand wohl weder keltisch sind, noch von Schwellbalken stammen, hatten die ersten 30 Jahre Forschung in der spätlatènezeitlichen Keltensiedlung den Beginn der Geschichte Basels endgültig vor die römische Eroberung gelegt. Doch als Vorgängerin der Stadt Basel sahen sie nur wenige an. Das Gros der Basler feierte sich noch 1957 zum 2000 Jahr-Jubiläum der Colonia Augusta Raurica als Erben der Römer. Erst seit der Entdeckung des Murus Gallicus, einer keltischen Befestigung auf dem Münsterhügel Anfang der 1970er Jahre sowie nach weiteren Ausgrabungen in der Siedlung am Rhein lässt sich der keltische Ursprung Basels nicht mehr wegdiskutieren. Das umfassende Auswertungsprojekt von Ludwig Berger und Andres Furger-Gunti in den 1970er Jahren macht «Basel-Gasfabrik» europaweit zu einer Referenzsiedlung. In den 1990er Jahren erlebt die Keltenforschung einen enormen Aufschwung: Galten die Kelten einst als tapfere, aber unzivili- sierte Barbaren, werden sie jetzt zu den Gründern Europas gekürt und das «Sandoz»-Areal zum Ursprungsort Basels. In der Barfüsserkirche präsentiert man stolz das «Gold der Helvetier». Doch wo viel Licht ist, da gibt es auch Schatten. Kritischen Warnungen zum Trotz wird «Basel-Gasfabrik» auf einer Linie mit Augusta Raurica ins mystische «Belchen-System» eingewoben.

Massive Baumassnahmen seit Ende der 1980er Jahre, zuerst auf dem damaligen «Sandoz»-Areal, dann im Bereich der Nordtangenten-Autobahn, danach auf dem Campus der Novartis und nun im Rheinhafen St. Johann, machten ganzjährige Rettungsgrabungen fast ohne Unterbruch nötig. Insgesamt sind mittlerweile mehr als $100000 \mathrm{~m}^{2}$ erforscht. Der Zeitdruck, zu retten, was möglich ist, fordert seinen Tribut. Eine Gesamtauswertung der Grabungen steht noch aus. Nicht zuletzt der Jahrhundertfund eines vollständig erhaltenen Depots im August 2010 bestätigt aber schon jetzt die These, dass «Basel-Gasfabrik» bereits zur keltischen Zeit ein bedeutendes Zentrum am Rhein war. -

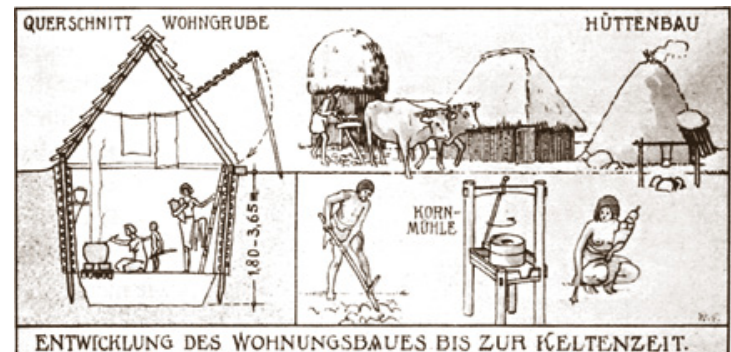

Abb. 18 Keltische Siedlung Basel-Gasfabrik, Rekonstruktionszeichnung von 1934. So stellte man sich damals die Siedlung und ihre Bebauung vor. Die Gruben wurden als Wohnhäuser betrachtet.

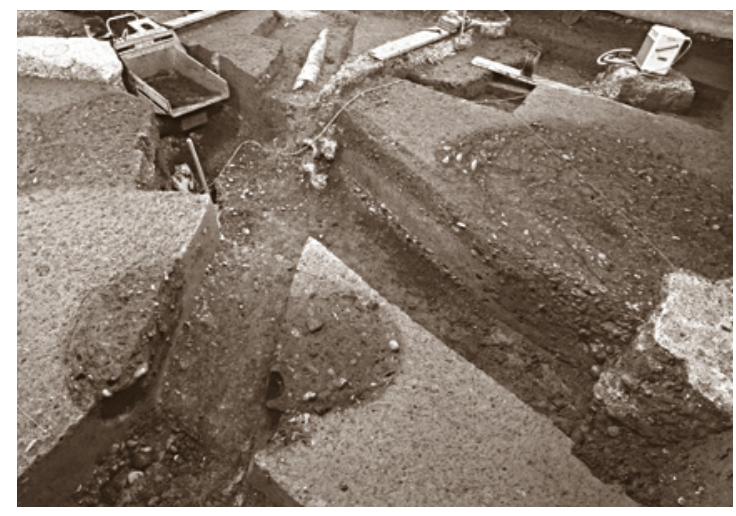

Abb. 19 Dunkel zeichnet sich rechts im Bild die Grube im Boden $a b$, in der später das Depot gefunden wurde. Foto: Michael Wenk. 


\section{AUSGRABEN MIT RÖNTGENBLICK}

Marion Benz

Um besonders kostbare und empfindliche Objekte zu bergen, werden sie samt der umgebenden Erde mit Gipsbinden stabilisiert und als handliche Blöcke entnommen. Im Computertomographen können sie dann geröntgt werden. Dieses medizintechnische 3D-Verfahren ermöglicht den Archäologen, Schicht für Schicht Form und Lage der Objekte genau zu betrachten. Die Röntgenstrahlen werden je nach Dichte des Objektes unterschiedlich stark durchgelassen. Bei besonders harten Gegenständen wie Metall prallen die Strahlen ab, diese Stellen sind auf dem Röntgenbild - ähnlich wie Knochen - einfach weiss. Je durchlässiger die Stoffe sind, um so weniger heben sie sich vom schwarzen Hintergrund ab. Mit Hilfe dieser Kontrastaufnahmen können die Archäologen und Restauratorinnen die Objekte viel gezielter freilegen. Die Gefahr, dass bei der Freilegung ein besonders stark korrodiertes Objekt zerstört wird, ist damit eingeschränkt, und selbst winzige Objekte wie kleine Perlen können präzise verortet und dokumentiert werden.

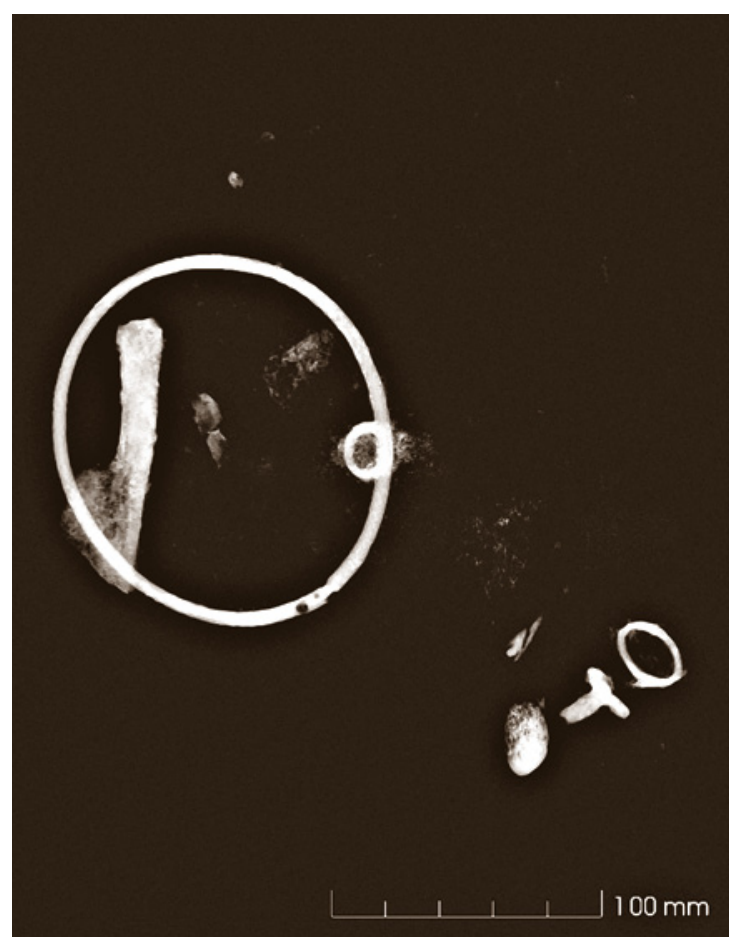

Abb. 20 Die Aufnahme des Computertomographen zeigt, dass sich neben dem grossen massiven Buntmetallring - oder sogar auf diesen aufgeschoben - ein weiterer kleiner Ring befindet. Deutlich ist auch der Steckverschluss des grossen Rings zu erkennen. Dabei ist noch fraglich, ob es sich um einen Halsring oder um ein so genanntes Steig- oder Steigergebiss eines Pferdezaums handelt.

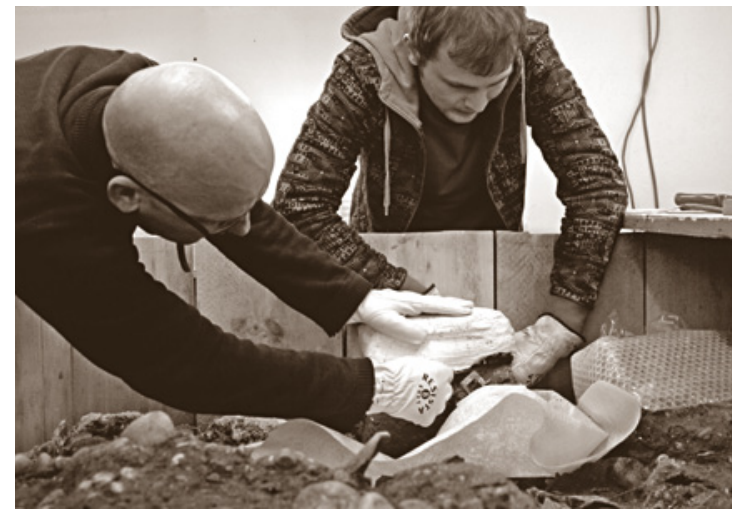

Abb. 21 Besonders fragile Stücke werden mit Gips ummantelt und als Block ins Universitätsspital Basel gebracht. Dort werden sie Schicht für Schicht im Computertomographen durchleuchtet. Foto: Julia Imhoof. 


\section{CHEMIE UND TECHNIK MÜSSEN STIMMEN}

Marion Benz

So sensationell der Jahrhundertfund des Depots ist, so gross sind die Herausforderungen, es zu bergen. Viele dünne, teilweise verzierte Buntmetallbleche zerfallen in kleinste Flitter. Auch die Holzobjekte, die sich in den trockenen Bereichen unter den Gefässen erhalten haben, sind so fragil, dass sie bei der leichtesten Berührung zerbröseln würden. Um die wertvollen Stücke in ihrer Lage präzise, aber dennoch zeitsparend zu dokumentieren und schonend zu bergen, setzen die Archäologen modernste Verfahren aus der digitalen Photographie und der Restaurierungstechnologie ein.

Informatiker haben ein Bildverarbeitungs-Programm entwickelt, das so genannte «Structure from Motion»-Verfahren, womit man aus vielen Aufnahmen aus unterschiedlichen Perspektiven ein massstabgetreues dreidimensionales digitales Modell erstellen kann. Diese Software ist zwar rechenintensiv, aber sie kann aus den zweidimensionalen Bildern die Raumtiefe er-rechnen und so die Lage jedes Objektes in Zusammenhang mit den anderen Objekten dokumentieren. Ohne aufwendige Zeichnungen und Messverfahren gelingt es den Archäologen so, die Lage der Objekte zueinander zumindest digital zu konservieren.

Damit die Objekte dann aber auch geborgen werden können, bedienen sich die Grabungstechniker und Restauratorinnen modernster Substanzen aus der Chemie. Bei ihren Forschungen haben Restauratoren chemische Stoffe gefunden, welche empfindliche Oberflächen wie die alter Gemälde für den Transport kurzfristig fixieren, dann aber spurlos wieder verschwinden. Das klingt unglaublich, aber in der Physik ist es ein bekanntes Phänomen: die sogenannte Sublimation. Es handelt sich um Stoffe, die direkt vom festen in den gasförmigen Zustand übergehen. Cyclododekan besitzt diese ungewöhnliche Eigenschaft und sublimiert bereits bei Zimmertemperatur. Erwärmt man Cyclododekan jedoch auf etwa $60 \mathrm{Grad}$, wird die Substanz flüssig, und man kann sie auf Objekte auftragen oder aufsprühen, die stabilisiert werden sollen. Sie kann aber auch in speziellen Lösungsmitteln oder in Treibgasen gelöst und dann sogar kalt verarbeitet werden.

Der grosse Vorteil: Im Gegensatz zu früher gebräuchlichen Härtern oder Füllmaterialien verflüchtigt sich Cyclododekan rückstandslos. Je nach Dicke des Auftrags und ob es als Lösung oder Sprühfilm aufgebracht wurde, verschwindet so die

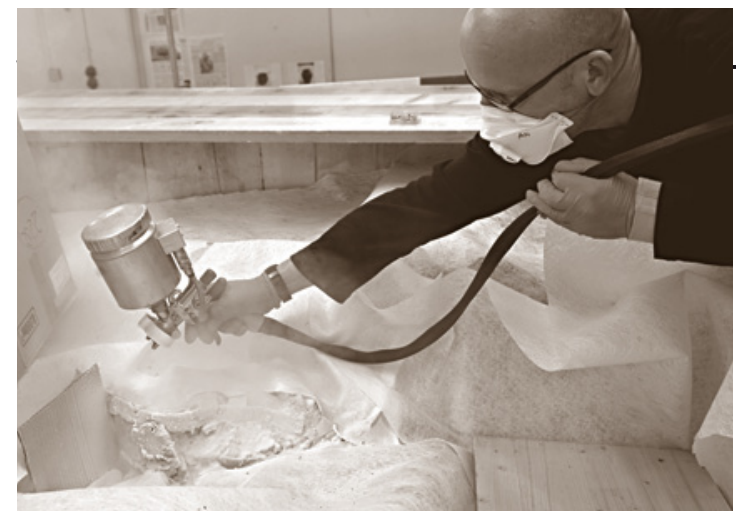

Abb. 22 Chemie im Dienste der Archäologie - mit Cyclododekan werden die fragilen Funde besprüht, um diese zu stabilisieren. Wie von Geisterhand verflüchtigt sich das wachsartige Mittel innerhalb weniger Wochen wieder rückstandslos. Dieser Sublimationsprozess kann durch Kühlen gebremst oder mit Heissluft gezielt gesteuert und punktuell beschleunigt werden. Foto: Michael Wenk.

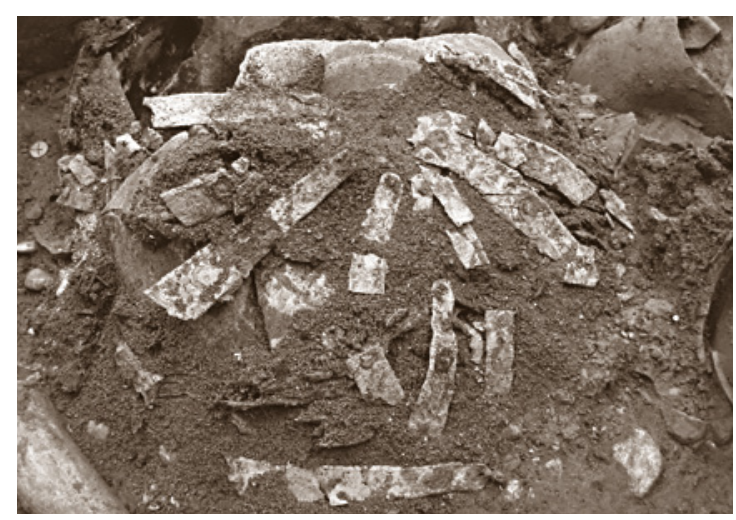

Abb. 23 Mega-3D-Puzzle mit Mikado-Effekt: Bei der Freilegung und Restaurierung muss jede Berührung der teils winzigen Bruchstücke wohl überlegt sein, da die keltischen Kostbarkeiten sehr fragil sind. Foto: Julia Imhoof. 